\title{
DISSERTATION
}

\section{Antenna Selection Diversity for IEEE 802.11p}

Conducted for the purpose of receiving the academic title

"Doktor der technischen Wissenschaften"

Mona Shemshaki, MSc.

born in Tehran, Iran, July $2^{\text {nd }}, 1980$

Matriculation number: 1128834

Aegidigasse 7-11/20, 1060 Vienna

November 23, 2016

Submitted to the TU Wien, Faculty of Electrical Engineering and Information Technology 

Advisor

Univ.Prof. Dipl.-Ing. Dr.-Ing. Christoph Mecklenbräuker TU Wien, Austria

Institute of Telecommunications

\section{Examiners}

Assoc. Prof. Dr. Javier Manuel Gozálvez Sempere

Miguel Hernandez University of Elche, Spain

Signal Theory and Communications

Prof. Dr.-Ing. Arrate Alonso Gómez

Mondragon Unibertsitatea, Spain

Signal Theory and Communications 

to my family 



\section{ABSTRACT}

In wireless vehicular communications, dependability plays an important role especially for the safety applications. Such requirement can be satisfied by multiple-input multipleoutput (MIMO) systems which are the-state-of-the-art in wireless communications. Although these systems provide spatial diversity and spatial multiplexing, they impose implementation costs due to the increased hardware and also software complexity. A low-cost feasible solution for MIMO systems is antenna selection diversity in which a subset of antenna elements is chosen for the transmitter and/or receiver.

In this work, I propose a receive antenna selection algorithm for vehicle-to-infrastructure (V2I) scenario in IEEE 802.11p. For this purpose, the IEEE 802.11p frame structure is extended by additional preambles. In the first part of the work, I introduce the antenna selection algorithm which allows to connect the antenna with the best instantaneous and predicted signal to noise ratio (SNR) to a single receive chain while maintaining the diversity gain. With the four added preambles to the frame structure, SNR estimation for each receive branch is feasible. In the next step, I discuss the receive antenna selection algorithm in combination with two different channel predictors with the same extended frame structure. Lagrange Interpolation and Linear Regression are the basis for the employed channel predictors.

In the second part, a measurement campaign is carried out in order to evaluate the performance of the antenna selection algorithm in an urban environment for V2I scenario. The Universal Software Radio Peripheral transceivers are used which combine graphical programming with flexibly configurable hardware.

Both simulation results and measurements data analysis results show that by employing antenna selection, higher diversity gain is achievable in a wireless vehicular network. Comparing to a single-input single-output system, there is a reduction in the bit/packet error ratio when antenna selection is used in a multiple antenna system. 



\section{KURZFASSUNG}

Für die drahtlose Vernetzung von Fahrzeugen hat die Verlässlichkeit eine hervorragende Bedeutung, insbesondere für sicherheitsrelevante Anwendungen. Die daraus entstehenden Anforderungen können durch den Einsatz von sogenannten multiple-input multipleoutput (MIMO) Systemen erfüllt werden, welche den Stand der Technik im Bereich der drahtlosen Kommunikation darstellen. Obwohl MIMO-Systeme räumliche Diversität bieten und den Einsatz von räumlichen Multiplexverfahren ermöglichen, generieren sie höhere Implementierungskosten aufgrund ihrer Hardware- und Softwarekomplexität. Eine preiswerte, realisierbare Lösung für MIMO-Systeme besteht in der Ausnutzung der Diversität durch Antennenauswahl, wobei nur ein Teil der Antennenelemente für den Sender und/oder Empfänger ausgewählt werden.

In dieser Arbeit schlage ich einen Algorithmus zur Auswahl von Empfangsantennen für Fahrzeug-zu-Infrastruktur (V2I) Kommunikationsszenarien mittels IEEE 802.11p vor. Hierfür wird die IEEE 802.11p Framestruktur um eine zusätzliche Präambel erweitert. Im ersten Teil meiner Arbeit stelle ich einen Algorithmus zur Auswahl von Empfangsantennen vor, der es erlaubt, die Antenne mit dem besten momentanen und geschätzten Signal-zu-Rausch Verhältnis (SNR) mit einer einzelnen Empfangskette zu verbinden, wobei der Diversitätsgewinn bewahrt wird. Durch die vier zur Framestruktur hinzugefügten Präambeln wird die SNR Schätzung für jeden Empfangszweig ermöglicht. Im nächsten Schritt erörtere ich einen Algorithmus zur Auswahl von Empfangsantennen in Verbindung mit zwei unterschiedlichen Kanalschätzern mit derselben Framestruktur. Lagrange Interpolation und Lineare Regression bilden die Grundlage für die eingesetzten Kanalschätzer.

Im zweiten Teil wird eine Messkampagne zur Bewertung der Performanz der Antennenauswahl Algorithmen in einer urbanen Umgebung für V2I Szenarien vorgestellt. Dafür werden Universal Software Radio Peripheral Transceiver eingesetzt, die grafisches Programmieren mit flexibel konfigurierbarer Hardware verbinden.

Sowohl Simulationsergebnisse als auch Ergebnisse der Messdatenanalyse zeigen, dass durch den Einsatz von Antennenauswahl ein höherer Diversitätsgewinn in drahtlosen Fahrzeugnetzen erreichbar ist. Im Vergleich mit einem single-input single-output System zeigt sich eine Verringerung der Bit- und Paketfehlerrate wenn Antennenauswahl in einem Mehrantennensystem eingesetzt wird. 



\section{ACKNOWLEDGEMENT}

First and foremost, I would like to express my profound gratitude to my advisor, Professor Christoph Mecklenbräuker, for giving me the opportunity to join his research group and pursue my education. Indeed, his continuous support, patience and motivation helped me all the time during my PhD years.

Besides my advisor, I am very grateful to Dr. Thomas Zemen for his support and interest in my research topic. Our scientific discussions and his insightful comments have widen my research perspective.

My sincere thanks also go to my colleagues who have been a source of not only scientific collaboration but also friendship; Mehdi Ashury and Markus Hofer, for their great help in carrying out the measurement campaign. Philipp Svoboda, Günther Koliander, Martin Müller, Gregor Lasser and Jelena Kaitović, for the fruitful discussions. Veronika Shivaldova and Levent Ekiz, for having memorable moments together during our trips. Sanda Drakulić, Geetha Ramachandran and Samira Homayouni, for always spreading good vibes to the working atmosphere.

I would like to express my special thanks to Mansoureh Tajaddod and Sina Larimian for always being there for me, particularly, in a very tough period of my life. Your friendship means a lot to me.

This work has been funded by the Christian Doppler Laboratory of Wireless Technologies for Sustainable Mobility. Moreover, I hereby would like to thank Austrian Institute of Technology GmbH (AIT) for providing the required equipment during the measurement campaign in Vienna.

Last but not least, my special thanks to my parents and my siblings for their unconditional love and encouragement which are invaluable. They have always supported me spiritually throughout my whole life especially during the last recent years, far in distance but near at heart. 



\section{CONTENT}

$\begin{array}{lll}1 & \text { Introduction } & 1\end{array}$

1.1 Wireless Vehicular Communications . . . . . . . . . . . . . . . . . . 1

1.2 MIMO Systems . . . . . . . . . . . . . . . . . . . . . . . . . . . . . . . . . . . . . . . .

1.3 Antenna Selection . . . . . . . . . . . . . . . . . . . . . . 4

1.4 Own Contribution . . . . . . . . . . . . . . . . . . . . . 6

1.5 Outline of the Thesis . . . . . . . . . . . . . . . . . . . 8

2 Antenna Selection Algorithm 9

2.1 System and Channel Model . . . . . . . . . . . . . . . . . . . 9

2.2 Preamble Design . . . . . . . . . . . . . . . . . . . . . . . . . . . . . . . . . . . . 10

2.3 SNR-based Antenna Selection Algorithm . . . . . . . . . . . . . . . 12

2.4 Channel Prediction-based Antenna Selection Algorithm . . . . . . . . . 13

2.4 .1 Lagrange Interpolation . . . . . . . . . . . . . . . . . . . . . . 14

2.4 .2 Linear Regression . . . . . . . . . . . . . . . . . . . . . . . . 15

2.5 Performance Evaluation . . . . . . . . . . . . . . . . . . . . . . . 16

2.6 Discussion and Conclusion . . . . . . . . . . . . . . . . . . . . . . 19

3 Antenna Selection Experiment with Software Defined Radio Design 23

3.1 LabVIEW Communications System Design Suite . . . . . . . . . . . . . 23

3.1 .1 IEEE 802.11 Application Framework . . . . . . . . . . . . . . 23

3.2 Antenna Selection Diversity Measurement Campaign . . . . . . . . . . 25

3.2 .1 Measurement Setup . . . . . . . . . . . . . . . . . 32

3.2.2 Data Analysis and Performance Evaluation . . . . . . . . . . . 34

3.3 Discussion and Conclusion . . . . . . . . . . . . . . . . . . . . . . . 44

4 Conclusions 47

4.1 Key Findings . . . . . . . . . . . . . . . . . . . . . . . . . . . . 47

4.2 Outlook . . . . . . . . . . . . . . . . . . . . . . . . . 48

\begin{tabular}{ll}
\hline Appendices & 51
\end{tabular}

A List of Terms and Symbols 53

B List of Acronyms 
C Measurement Campaign Testbed 57

C.1 PXle-1082 . . . . . . . . . . . . . . . . . . . . . . . . . . 57

C.2 USRP-2953R . . . . . . . . . . . . . . . . . . . . . 60

$\begin{array}{ll}\text { Bibliography } & 63\end{array}$ 


\section{LIST OF FIGURES}

1.1 Vehicular System Functionality [1] . . . . . . . . . . . . . . 2

1.2 Receive Diversity [2] . . . . . . . . . . . . . . . . . . . . . . . . 5

1.3 A generalized selection diversity system [2] . . . . . . . . . . . . 6

2.1 IEEE 802.11p Physical Layer (PHY) Packet Structure [3] . . . . . . . . 10

2.2 IEEE 802.11p OFDM Pilot Structure [4] . . . . . . . . . . . . . . 11

2.3 Modified Frame Structure [5] . . . . . . . . . . . . . . . . . . . 11

2.4 Matrix of estimated SNR and selected index $\hat{n}$ according to (2.5) . . . 12

2.5 Antenna selection performance with Lagrange Interpolation . . . . . . . 18

2.6 Antenna selection performance with Linear Regression . . . . . . . . . . 19

2.7 Antenna selection with channel predictor and SISO . . . . . . . . . 20

2.8 Antenna selection performance of channel predictors . . . . . . . . . 21

3.1 Functional split between Host, FPGA and RF . . . . . . . . . . . 25

3.2 Added OFDM symbol duration for IEEE 802.11p . . . . . . . . . . . 26

3.3 Added sample rate for IEEE 802.11p at Tx . . . . . . . . . . . . . 27

3.4 Added sample rate for IEEE 802.11p at RX . . . . . . . . . . . . . . 28

3.5 Decision making process of the antenna selection algorithm . . . . . . . 29

3.6 Measurement track for direct path . . . . . . . . . . . . . . . . 30

3.7 Measurement track for L-Turn . . . . . . . . . . . . . . . . . . . 31

3.8 Measurement track for Under Subway Path . . . . . . . . . . . . . . . 31

3.9 Omnidirectional Antenna as $\mathrm{Tx} \ldots \ldots . \ldots . . \ldots 33$

3.10 Antenna mounted at a fixed position as $\mathrm{Tx} \ldots . . \ldots 33$

3.11 Antennas mounted on the roof of the vehicle as $\mathrm{Rx} \ldots \ldots . . . . .34$

3.12 USRP-2953R [6] . . . . . . . . . . . . . . . . . . . . . . . . . . . . . 34

3.13 PXle-1082 (PXI Express chassis) [7] . . . . . . . . . . . . . . . . 34

3.14 SISO - Direct path - Total distance . . . . . . . . . . . . . 37

3.15 SISO - Direct path - Distance from RSU . . . . . . . . . . . . . . 38

3.16 Antenna selection - Direct path - Total distance . . . . . . . . . . . . 39

3.17 Antenna selection - Direct path - Distance from RSU . . . . . . . . . 40 
$3.18 \mathrm{SISO}$ - L-Turn - Total distance . . . . . . . . . . . . . . . . . 40

3.19 SISO - L-Turn - Distance from RSU . . . . . . . . . . . . . . . . 41

3.20 Antenna selection - L-Turn - Total distance . . . . . . . . . . . . . 41

3.21 Antenna selection - L-Turn - Distance from RSU . . . . . . . . . . . . 42

3.22 SISO - Subway - Total distance . . . . . . . . . . . . . . . . . . 43

3.23 SISO - Subway - Distance from RSU . . . . . . . . . . . . . . . . 43

3.24 Antenna selection - Subway - Total distance . . . . . . . . . . . . . . 44

3.25 Antenna selection - Subway - Distance from RSU . . . . . . . . . . . . 45

C.1 PXle-1082 Backplane Overview . . . . . . . . . . . . . . . . . . 57

C.2 PXle-1082 Chassis Dimensions (front and side) . . . . . . . . . . 58

C.3 PXle-1082 Chassis Dimensions (bottom) . . . . . . . . . . . . . . . 59

C.4 PXle-1082 Chassis Rack Mount Kit Components . . . . . . . . . . . . 59

C.5 USRP RIO Block Diagram . . . . . . . . . . . . . . . . . . . . 61

C.6 USRP RIO System Component . . . . . . . . . . . . . . . . . . . . . . 61 


\section{LIST OF TABLES}

2.1 Simulation Parameters $\ldots \ldots \ldots$. . . . . . . . . . . . . . . 16

3.1 Transmit antenna location for each scenario . . . . . . . . . . . . 30

3.2 Physical parameters for the transmitter and receiver antennas . . . . . . 32

3.3 Measurement parameters . . . . . . . . . . . . . . . . 32 



\section{INTRODUCTION}

In this chapter, there is an overview of the wireless vehicular communications and the motivation for this field in the research activities. Since the main focus of my thesis is on antenna selection diversity in the vehicular environment, afterwards, related works to this topic are discussed. In the last section of this chapter, I will briefly introduce my contribution that has been done in this area following by the outline of the rest of the thesis.

\subsection{Wireless Vehicular Communications}

Recently, wireless vehicular communications have received a significant interest not only from academia but also from industry due to the urge to reduce the traffic congestion and vehicle accidents and many technologies have been employed to assist and also manage the transportation. Vehicle-to-vehicle/infrastructure (V2X) communications have the potential to enhance the road safety and the traffic efficiency and also reduce emissions, therefore, this technology plays an important role in the future of Intelligent Transport System (ITS) [8]. The vehicles can be provided with computational and communication platforms in order to improve the sensing capabilities and moreover, they can be an assistant to the driver and also can enhance transportation safety for human being. In addition to the enhancement of safety and traffic efficiency, wireless communications can also provide infotainment applications that can bring commercial value for the owner and also the manufacturer of the vehicle [9]. In the early 90 s, the main research topic was the development of an intelligent vehicle highway systems (IVHSs) [10]. The idea of developing IVHS has not happened yet but V2X communications have being developed. As shown in Fig. 1.1, in a vehicular communication system, the vehicles and Road Side Unit (RSU) contribute with their relevant information to provide a collaborative environment. The vehicles can avoid dangerous situations according to the received information from RSU and also from the neighboring vehicles. Examples of such unwanted or dangerous situations which can be detected or anticipated by a vehicle are shown in the mentioned figure.

IEEE 802.11 is a commercial off-the-shelf (COTS) technology to be used in the V2X communications, so, the vehicles and the infrastructure use an IEEE 802.11-based wireless network interface to create a network and this network is the mean to communicate with each other for the safety applications. Such communications include cooperative collision warnings due to a seen accident or the emergency warning messages to lower the vehicle's speed based on the brake light of the fronts vehicles. 


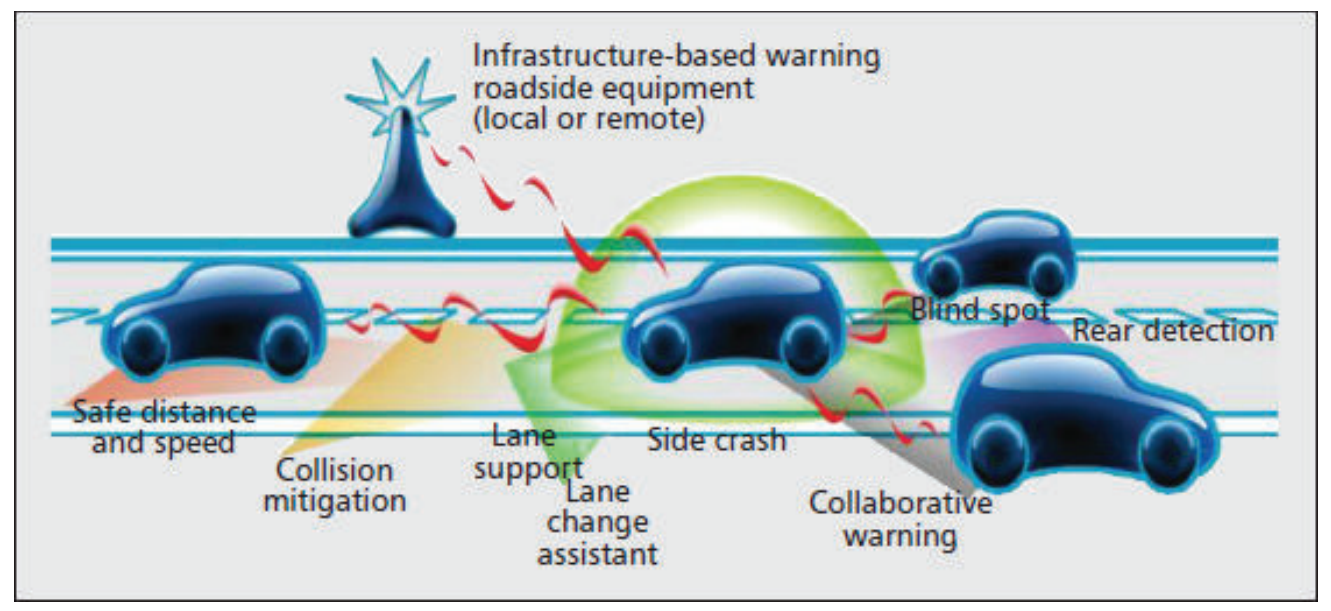

Figure 1.1: Vehicular System Functionality [1]

Major challenges regarding the transportation design are to reduce the occurrence of accidents and to lighten the traffic congestion [11]. The goal to have a safer, more efficient and environmentally friendly transportation has resulted in many research topics which have being intensively studied and investigated in the recent years. For this purpose, dependable wireless vehicular communications are required in order to provide robust connectivity [12] and [13]. Dependable connectivity plays an important role in ITS which means that the vehicular communication links are reliable with low-latency and they are capable of handling strict packet delay deadlines. In ITS, V2I and Vehicleto-Vehicle $(\mathrm{V} 2 \mathrm{~V})$ communications are both needed. The reason is that the gathered data from the road users are exchanged among the road users and also with the road infrastructure.

One of the challenging safety applications is the intersections which are complex and dangerous driving environments [8]. The INTERSAFE project has been developed in Europe in order to enhance safety at the intersections by combining sensor and communications technology [14]. In addition to Europe, this safety application has been an appealing research topic in Japan and US as well under the project names of the Driving Safety Support Systems (DSSS) [15] and the Cooperative Intersection Collision Avoidance Systems (CICAS) [16], respectively.

The used cases of safety application in intersections have been described in [8]. Such use cases include warning about the traffic light status via V2X communications and also the remaining time until its next change, warning about turning and crossing path collision, warning about rear-end collision which is about an accident due to sudden break of a vehicle at an intersection, traffic efficiency in which RSU monitors the intersection's traffic.

\subsection{MIMO Systems}

MIMO systems have recently obtained great interest in the wireless communication systems. In such system, multiple antenna elements are used at both link ends, trans- 
mitter and receiver. MIMO systems are able to provide higher capacities than conventional single-input single-output (SISO) systems by sending several parallel data streams. Therefore, in applications where high capacity is required, such as hotspots, employment of MIMO systems is suitable because this technology takes advantage of multipath effect and high capacity can be achieved [17]. The very first studies that shows the large capacities of MIMO systems with simulation results were done in 1980s [18] and later on the capacity of MIMO systems were analytically investigated [17], [19].

In addition to the increase in channel capacity, MIMO technology can also result in more reliable transmission and can provide an antenna diversity system that can combat the fading's effects. In such system, the diversity is achieved by transmitting multiple copies of one signal which are independent and can be combined into a high quality. The advantage is that in case there are some low quality copies of the signal, there would be still possibility to have a high quality signal [20].

For more than 50 years, the antenna diversity at the receiver side has been a research topic. In such system, there are $M_{r}$ receive antenna elements at the receiver and the diversity order is also equal to $M_{r}$. Diversity order shows how effective the diversity is so that the deep fades are avoided. The slope of the bit/packet error ratio (BER/PER) vs. SNR curve is corresponding to the diversity order. In [21], the diversity order $d$ is defined as in (1.1) for a system that has an average error probability $\bar{P}_{e}(\mathrm{SNR})$ as a function of SNR. By means of multiple antennas at the receiver the mean SNR is also improved. On the other hand, the antenna diversity at the transmitter side has been started since 1990s and comparing to the receiver diversity is a more recent research topic [20]. For the transmit diversity, the channel is either know or unknown. In case the channel is known, the gains are the same as receiver diversity and if the channel is unknown, high diversity gain is achieved but the average SNR has no improvements. The last option would be to combine transmit and receive diversity which will result in an $M_{t} M_{r}$ diversity order [20].

$$
\lim _{\mathrm{SNR} \rightarrow \infty} \frac{\log \left(\bar{P}_{e}(\mathrm{SNR})\right)}{\log (\mathrm{SNR})}=-d
$$

As mentioned above, a MIMO system can also be used to obtain spatial multiplexing which is the transmission of different data streams in parallel from different transmit antennas. At the receiver side, the different data streams are separated by multiple receive antennas. In such system, there is $M_{r}$ combinations of $M_{t}$ transmitted signals which can be linearly independent in case of a well-behaved channel. By this method the data rate can be increased by a factor of $M_{t}$ while there is no need for additional spectrum and more power expenditure.

Additionally, by exploiting MIMO system, the interference reduction can be designed if the channel state information (CSI) of the users are known. In case the CSI is available, the interference energy that is sent to neighbors other than the intended receiver is minimized and as a result, the interference would be less [22].

An extended MIMO is the massive MIMO technology which is a multi-antenna system 
with a large number of antennas that aims to improve the spectral efficiency as well as the power efficiency, remarkably [23] and comparing with the conventional MIMO systems, massive MIMO system can even bring the MIMO benefits more to the system. It should be noted that in such system, due to the large number of the transmit and receive antennas, the hardware cost is quite prohibitive [24].

There is no doubt that MIMO systems and vehicular adhoc networks (VANET) have been investigated separately and are different research topics but employing MIMO systems in vehicular communication would be beneficial. In [22], the challenges and opportunities of MIMO systems in VANETs are discussed. Due to the diversity gain in MIMO systems, in safety applications of the vehicular communications where reliable communications play an important role, MIMO systems are able to offer such communications in VANETs. Moreover, in the vehicular environment with high mobility, the diversity and spatial multiplexing gain of MIMO systems can be an asset. In addition to safety applications of VANETs, there are infotainment applications and services which involved with mobile videos and for sure comparing the high data rate of MIMO with SISO systems, using MIMO systems in vehicular environments can support such applications. Furthermore, the transmission delay of warning messages in safety applications would be minimized by means of the MIMO systems.

\subsection{Antenna Selection}

Although there are advantages of exploiting a MIMO system as described in section 1.2. there would be always some considerations for such systems regarding the complexity and indeed the cost. By having additional antennas, there will be increase in the antenna elements, digital signal processing and Radio Frequency (RF) elements which among them, the antenna elements and processing units are cheap while RF elements are expensive. Moreover, in a MIMO system with $M_{t}$ transmit antennas and $M_{r}$ receive antennas, $M_{t}$ and $M_{r}$ complete RF chains exit at the transmitter and the receiver, respectively. Therefore, in order to combat the mentioned drawbacks, the antenna selection schemes have attracted great attention in which $N_{t}$ or $N_{t r}$ out of $M$ antennas are chosen. Such selection can be either at both link ends or just at one side. By such approach, the number of RF chains is reduced and consequently, the high hardware cost will be decreased. Thus, it means that by having antenna selection approach, the cost can be alleviate and at the same time, obtain the advantages of MIMO systems.

It should be noted that by exploiting MIMO system and the antenna selection scheme in the wireless vehicular communications, the main obstacle would be regarding the channel. Due to the time-varying channel in vehicular environment, the antenna selection requires additional preambles for the channel estimation and prediction, therefore, the channel will be changed.

Since in my work, the proposed antenna selection algorithm is at the receiver side, here the receive antenna selection is described. In a diversity reception system, there are multiple versions of a transmitted signal with different fading coefficient and noise at the receiver side as shown in Fig. 1.2. In order to have diversity, the received signals should be combined. There are three different methods to perform the diversity combining; 


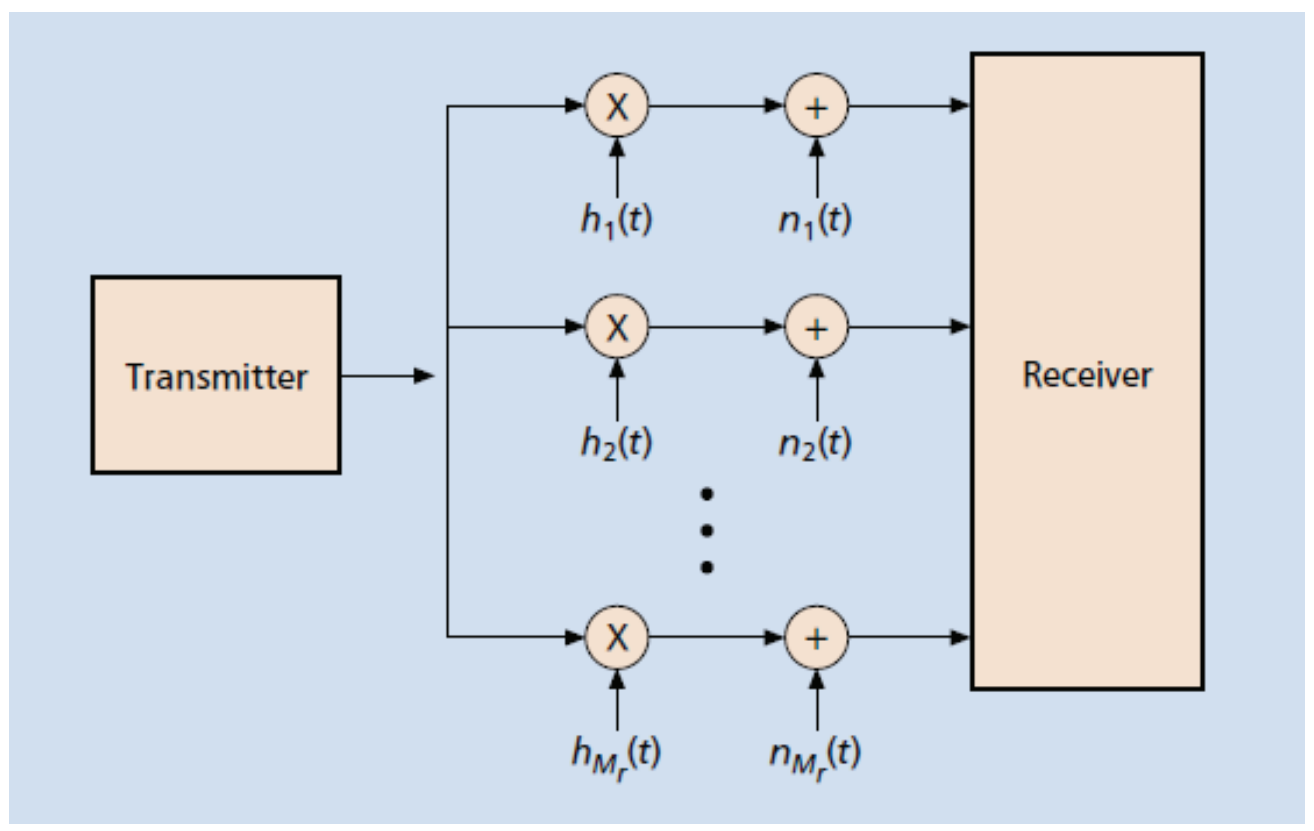

Figure 1.2: Receive Diversity [2]

Selection diversity, maximal ratio combining (MRC) and equal gain combining (EGC). In selection diversity method, the path with the highest SNR is chosen while in MRC method, the decision is made based on an optimal linear combination of the path signals and finally, in EGC method, the path signals will be added after being co-phased.

In a receive antenna selection scheme, a subset of the receive antennas are chosen and their signals will be combined, accordingly. This is the generalized selection which is illustrated in Fig. 1.3 and also known as hybrid selection. After a subset of receive antennas are chosen, the selected path signals can be combined by either MRC or EGC [2].

Although receive antenna selection provided diversity gain while reducing the hardware cost of multiple antennas, it is required to have the perfect channel knowledge and a slowly time-varying fading channel. In [25], the authors have proposed a receive antenna method which employs discrete prolate spheroidal (DPS) sequences that required only the Doppler bandwidth knowledge and no further need to have detailed knowledge of correlation. The proposed method can accurately estimate and predict the time-varying frequency flat channels.

There are three impediments in most of the works in the area of antenna selection. The first one is the assumption that the pilot symbols are divided equally by the number of antenna elements, in other words, equal allocation. Such assumption is not optimal for slowly-varying channels. Second one is about the packet reception and the possibility to obtain additional information on the channel from the link-layer error checks which is typically missed in the literature. The last drawback is regarding the channel model which is usually a quasi-static block-fading model. Such model prevents the receiver 


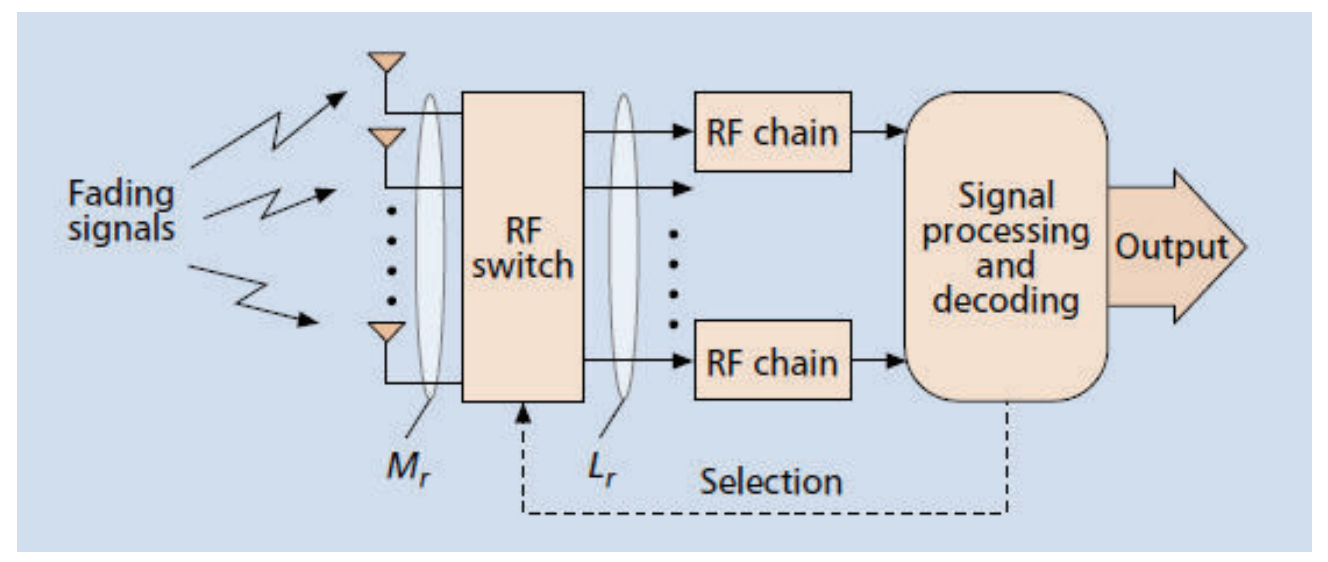

Figure 1.3: A generalized selection diversity system [2]

from fully using the temporal channel correlation. A Markov decision approach for pilot allocation and also receive antenna selection is presented in [26] that have considered the mentioned drawbacks.

In [27], an adaptive antenna selection technique has been presented which can increase the throughput while the system is energy efficient. In this study, the selection has been performed at the transmitter side since with a fixed number of transmit antennas, achieving better throughput and energy efficiency is not feasible.

The receive antenna selection is more common than the transmit antenna selection because for the latter scheme, the channel state information or the receiver knowledge is required at the transmitter. Usually, this knowledge is provided at the transmitter side by data transmission in the reverse direction which will impose a delay to the system and accordingly the outdated knowledge can effect the system performance. One solution would be to employ a prediction scheme. In this scheme, the prediction has been done based on the past CSI to predict for the current data frame [28].

A transmit antenna selection scheme along with a receiver maximal-ratio combining has been studied in a MIMO system with flat Rayleigh fading channel [29] in which a subset of antenna elements are selected at the transmitter and at the receiver. The selection criterion is the maximum of the total received signal power. There is a feedback channel which informs the transmitter about the selected antenna.

\subsection{Own Contribution}

Due to the mentioned challenges of MIMO systems and at the same time because of their advantages in wireless vehicular communications, I have focused on proposing an antenna selection algorithm which can be a feasible solutions to reduce the high cost imposed by multiple RF chains and in addition to achieve higher diversity gain which means a more reliable system.

In this thesis, I present an antenna selection algorithm for wireless vehicular communi- 
cations which improves the diversity gain. The proposed algorithm is based on IEEE 802.11p with modifications in the frame structure. The difference is in the number of the preambles. Four preambles have been added at the beginning of the frame, so, instead of one preamble as in IEEE 802.11p, there are five preambles and four of them are used for the antenna selection purpose.

The performance of the proposed algorithm has been evaluated through numeric Monte Carlo simulations and also has been tested in real environment during a measurement campaign. Obtained results from both have confirmed the efficiency of employing an antenna selection algorithm in the vehicular environment.

The list of publications are as follows:

- M. Shemshaki, T. Zemen, C. Mecklenbräuker, "Antenna Selection Diversity for IEEE 802.11p", in Proc. of 2013 IEEE 39th Annual Conference of Industrial Electronics Society (IECON), Vienna, Austria, November, 2013.

- M. Shemshaki, C. Mecklenbräuker, "Antenna Selection Algorithm with Improved Channel Predictor for Vehicular Environment", in Proc. of IEEE 6th International Symposium on Wireless Vehicular Communications (WiVec), Vancouver, Canada, September, 2014.

- M. Shemshaki, G. Lasser, L. Ekiz, C. Mecklenbräuker, "Empirical Path Loss Model Fit from Measurements from a Vehicle-to-infrastructure Network in Munich at $5.9 \mathrm{GHz}$ ", in Proc. of 2015 IEEE 26th Annual International Symposium on Personal, Indoor, and Mobile Radio Communications (PIMRC), Hong Kong, Hong Kong, August-September, 2015.

- M. Shemshaki, Mehdi Ashury, Markus Hofer, T. Zemen, C. Mecklenbräuker, "Evaluation of Antenna Selection Diversity for IEEE 802.11p by Packet Error Measurements in Urban Areas", to be submitted to IEEE Transactions on Vehicular Technology.

Additionally, the contributions to COST Action IC1004 on "Cooperative Radio Communications for Green Smart Environments" are listed as following temporary documents (TD):

- COST IC1004 TD(12)05008 M. Shemshaki and C. Mecklenbräuker: Empirical Pathloss Models Applicable for LTE Systems in Wireless Vehicular Communications, presented at 5th Management Committee Meeting, Bristol, UK, 24-26 September 2012. .

- COST IC1004 TD(14)10059 M. Shemshaki and C. Mecklenbräuker: Antenna Selection Scheme with Channel Prediction for IEEE 802.11p, presented at 10th Management Committee Meeting, Aalborg, Denmark, 26-28 May, 2014. 


\subsection{Outline of the Thesis}

This thesis is written based on the above mentioned publications, the ones mainly focused on the antenna selection algorithm. The content of the each individual chapter and the main contributions of the thesis are briefly described as follows:

Chapter 1 introduces the wireless vehicular communications and the advantages of such kind of system in the transportation. Then, MIMO systems are explained and describing the benefits that are provided with the employment of such systems. On the other hand, the remarkable disadvantage of these systems is explained and as a feasible solution to these drawbacks, antenna selection algorithm is described.The last section of the chapter provides the outline of the thesis and summarizes the content of the individual chapters.

Chapter 2 proposes an antenna selection algorithm for IEEE 802.11p that can provide higher diversity gain for vehicular scenarios. In this chapter, first, the proposed frame structure is described which has four additional preambles comparing to the one in IEEE 802.11p. Afterwards, two different antenna selection algorithms are presented in detail; the first algorithm is based on estimated SNR values and the second algorithm has the channel predictor in order to select the better link for transmission. In both approaches, a system including one transmitter and two receivers is considered. In the last part of this chapter, the simulation results are shown in order to evaluate the performance of the proposed algorithms. Monte Carlo simulations are used for the evaluation purpose.

Chapter 3 investigates the performance of the proposed algorithm in real environment. This chapter consists of two main sections; the first one describes the LabView Communications System Design Suite and the IEEE 802.11 application framework in which the proposed antenna selection algorithm is implemented and the required modifications are applied and the second part explains the experiments that have been carried out in Vienna, Austria to test the algorithm. At the end of the latter section, the data analysis results are shown and the performance of the algorithm is evaluated via the captured data during the measurement.

Conclusions chapter sums up the research which has been done and shows the remarkable achievements by using the proposed algorithm in vehicular environments.

Appendix presents the list of terms and symbols and additionally, the list of acronyms. Furthermore, it provides the list of references which have been cited through this thesis. 


\section{ANTENNA SELECTION ALGORITHM}

This chapter defines the antenna selection algorithm which was studied throughout the thesis. First, the system and channel model are explained and then, the extended frame structure which is proposed for the purpose of antenna selection is presented. The proposed frame structure with additional preambles is a modified version of the one introduced in IEEE 802.11p. In the last section of this chapter, the channel prediction methods which are used to improve the antenna selection performance are discussed in detail and also the performance of the proposed algorithm is evaluated by means of the simulation results.

\subsection{System and Channel Model}

In my work, the system model is a single-input multiple-output (SIMO) system that has one transmit antenna $\left(M_{t}=1\right)$ and $M_{r}$ receive antennas from which a subset of optimum receive antennas $N_{r}$ are selected for reception. For each link between transmitter and receiver, the time-variant frequency response $H_{n}(t, f)$ is defined, where $n$ denote the receiver index, $t$ denotes time and $f$ corresponds to the frequency.

The proposed algorithm for antenna selection diversity is tailored to a MIMO-OFDM system. The Orthogonal Frequency Division Multiplexing (OFDM) system has $N$ subcarriers and a cyclic prefix of length $G$. The transmission unit is a frame with a frame length of $L$ OFDM symbols. The system utilizes a bandwidth of $B$ and a sampling rate at the receiver side $B=1 / T_{C}$ [4]. We assume the cyclic prefix is longer than the maximum path delay, such that the sampled time-variant frequency response can be rewritten as below, [30]:

$$
H_{n}[p, q]=H_{n}\left(p T_{S}, q \Delta f\right)
$$

where $p \in\{0, \ldots, L-1\}$ denotes the OFDM symbol index, $q \in\{0, \ldots, N-1\}$ the subcarrier index, $T_{S}=1 / B(N+G)$ the OFDM symbol length and $\Delta f=B / N$ the subcarrier spacing.

After the OFDM demodulation the received signal can be expressed as in (2.2), [30] 


$$
Y_{n}[p, q]=\sum H_{n}[p, q] X[p, q]+\eta_{n}[p, q]
$$

where $X[p, q]$ denotes the symbols sent from the transmit antenna and $\eta_{n}$ denotes additive white Gaussian noise at the $n$-th receive antenna.

\subsection{Preamble Design}

The proposed antenna selection algorithm is based on the preamble symbols and by exploiting the training symbols of preamble, the "better" link for transmission is chosen. It should be noted that several definitions of "better" are possible. Therefore, in this section, first, the OFDM frame and pilot structures for IEEE.802.11p are explained as the major basis for our antenna selection. Then, the modified frame structure is presented which is an extension of the frame and pilot structure of IEEE 802.11p.

In Fig. 2.1, the IEEE 802.11p frame structure is illustrated. As it is shown, a frame consists of 12 preamble symbols in which 10 short training symbols and 2 long training symbols exist. Each preamble symbol duration is $16 \mu \mathrm{s}$. The next symbol in the frame is the SIGNAL symbol which is equal to one OFDM symbol and after that there are variable number of OFDM symbols that are assigned to DATA. As it is shown in this figure, there is a Physical Layer Convergence Protocol (PLCP) header in this structure which consists of different parts. The first part of this header is RATE which shows the data rate. Another part of this header is shown as LENGTH that indicates the number of microseconds required to transmit Physical Layer Service Data Unit (PSDU).

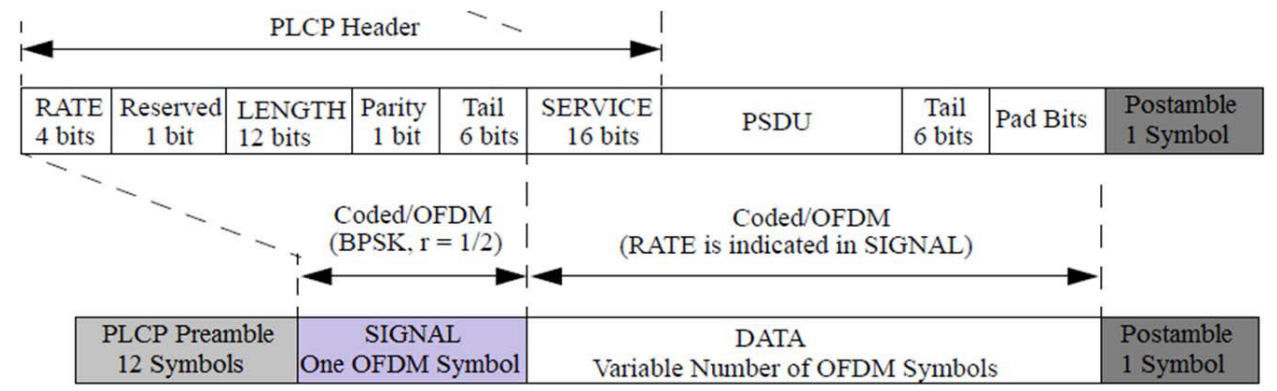

Figure 2.1: IEEE 802.11p Physical Layer (PHY) Packet Structure [3]

Fig. 2.2 shows the pilot structure in IEEE 802.11p OFDM frame. As it is represented in this figure, the first two columns are the long preamble symbols which are used for channel estimation and afterwards the SIGNAL and DATA OFDM symbols are placed. It should be noted that the short preamble has not been shown in this figure. At the end of the frame, one postamble symbol has been considered in the reserved bit of the 
header structure [4]. In the frequency domain, four pilots have been combed through the whole OFDM frame and they are employed to compensate the carrier frequency offset.

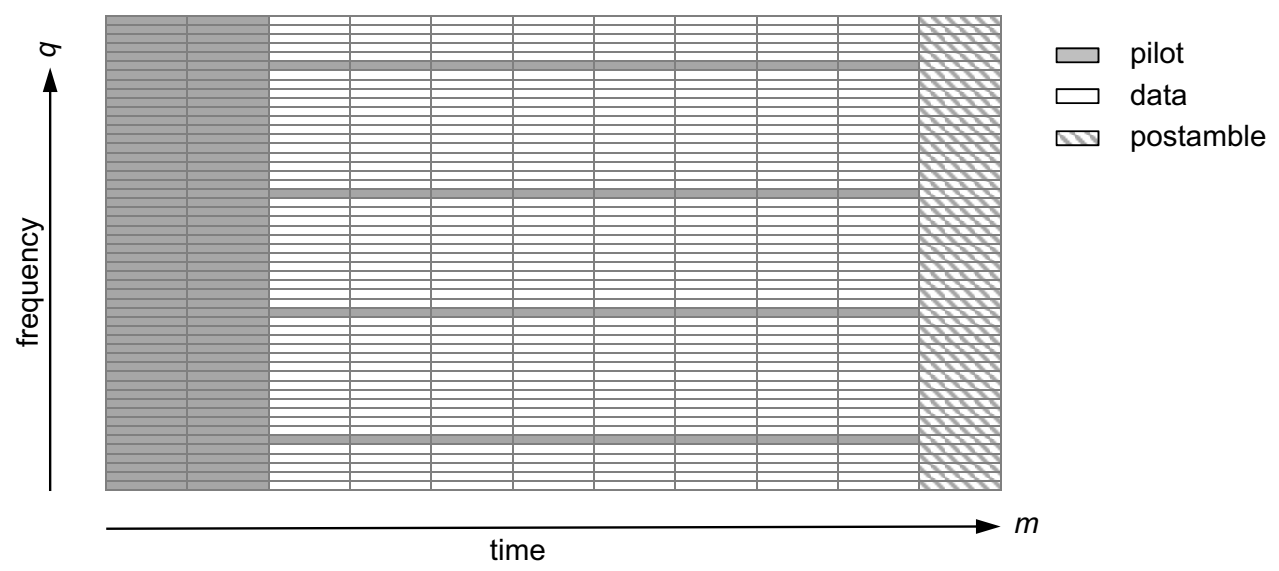

Figure 2.2: IEEE 802.11p OFDM Pilot Structure [4]

As mentioned above, the antenna selection algorithm is carried out based on the training symbols and for this purpose, the above illustrated frame structure is modified and four more preambles are added to the current IEEE $802.11 \mathrm{p}$ frame. The modified frame structure is shown in Fig. 2.3 in which $M_{t}=1$ and $M_{r}=2$. At the receiver side, there is switching between the receive antennas during the selection phase. As illustrated, the first added preamble is transmitted and received by the first receive antenna $(R \times 1)$ and with a $16 \mu$ s time interval, the second added preamble is sent and received by the second receive antenna $(R \times 2)$. Same process is carried out for the third and fourth added preambles, as well. Then, after a delay of $16 \mu \mathrm{s}$, the data frame is transmitted to the selected receive antenna. The $16 \mu \mathrm{s}$ is the time which is required to switch between the receiver antennas. Each preamble consists of the short and long training symbols which is denoted by $\mathrm{T} 1$ and $\mathrm{T} 2$ in this figure. The $16 \mu$ s delay which is considered in this work, is a feasible switching time.

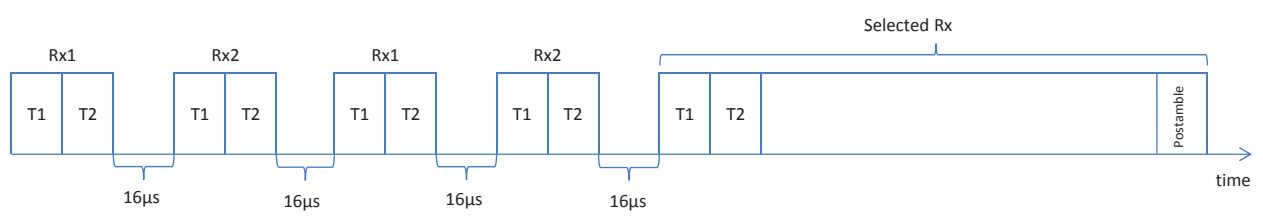

Figure 2.3: Modified Frame Structure [5] 


\subsection{SNR-based Antenna Selection Algorithm}

As described in section 2.2, the added preambles are received by receive antennas; two added preambles per receive antenna. At each receive antenna, the SNR is estimated for each subcarrier as written in (2.13) and based on the estimated SNR values for all subcarriers, a matrix is formed which contains the estimated values for all possible switching states of the transmit antenna and receive antennas, which in this work is two, since there is one transmit antenna and two receive antennas. In Fig. 2.4 the matrix is shown.

$$
\widehat{\operatorname{SNR}}_{n}[q]=\frac{\hat{S}_{n}[q]}{\sigma_{N}^{2}}, \quad(n=1,2 ; q=1, \ldots, N)
$$

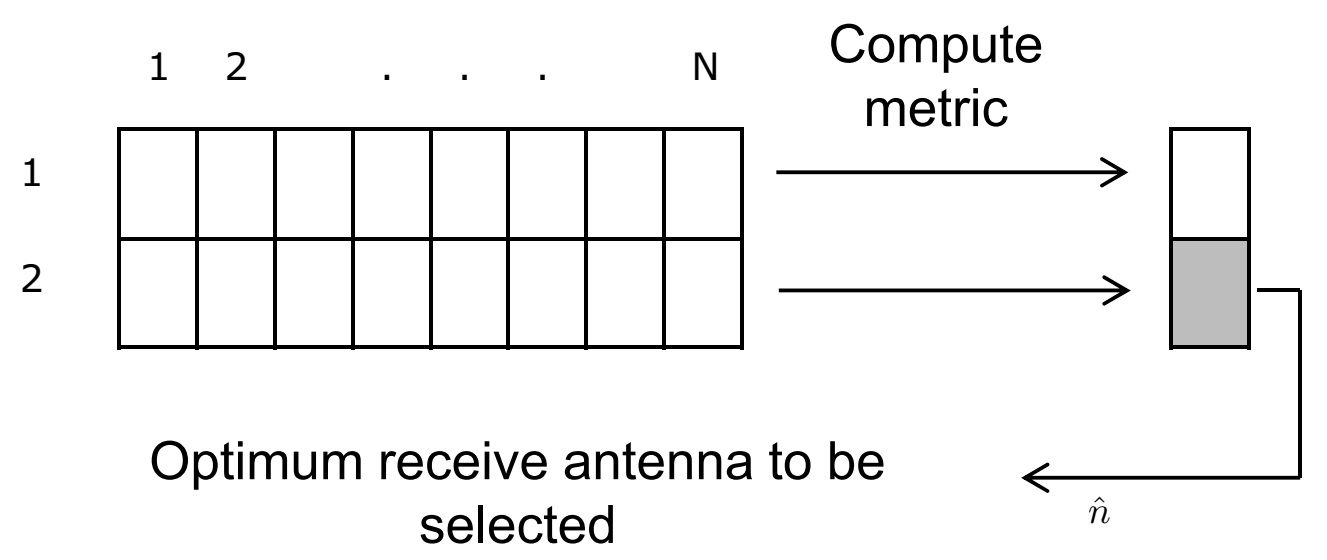

Figure 2.4: Matrix of estimated SNR and selected index $\hat{n}$ according to (2.5)

The $(n, q)$-element of the matrix stores the estimated SNR for receive antenna $n$ and subcarrier $q$,

where $\sigma_{N}^{2}$ is the noise variance which is considered constant in the simulation and the estimated signal power $\hat{S}_{n}[q]$ is evaluated as:

$$
\hat{S}_{n}[q]=\sum_{p=1}^{2}\left|Y_{n}[p, q]\right|^{2}
$$

We note that $\widehat{\mathrm{SNR}}_{n}[q]$ is evaluated from the $n$th transmitted preamble. The number of rows in this matrix is $\left(\begin{array}{l}2 \\ 1\end{array}\right)=2$ which equals the number of all switching states. It has $N$ columns corresponding to the number of subcarriers. We choose the following maxmin criterion for antenna selection 


$$
\hat{n}=\arg \max _{n=1,2}\left(\min _{1 \leq q \leq N} \widehat{\mathrm{SNR}}_{n}[q]\right)
$$

The minimization is over subcarriers and the maximization is over all combinations of antenna pairs. Therefore, first in each row of this matrix, the minimum value of the SNR is chosen and then a vector is formed which contains these minimum values and consequently the maximum SNR value is selected in this vector which leads to the optimum receive antenna since the rows of the vector correspond to the receive antennas. In other words, by using the maxmin function, among worst cases which is the minimum SNR in this work, the best one is chosen.

\subsection{Channel Prediction-based Antenna Selection Algorithm}

In wireless vehicular communications, the channel is time-varying due to the mobility of the vehicles and the channel state information is rapidly outdated, thus, I have proposed to employ the channel predictor in order to have a better performance of the antenna selection algorithm. In this section, the channel predictors which are used in my work are explained.

For the purpose of channel prediction, it is required to have the channel estimation of the system. Therefore, in the training phase, first, the channel estimation is done for the additional preambles and then, the prediction is carried out for the fifth preamble which is at the beginning of the frame. By using a Least Square (LS) estimator, the channel coefficients are estimated at each receive antenna for the added preambles, as written in (2.6).

$$
\hat{\mathbf{H}}_{n}[p, q]=\left(\mathbf{X}^{H}[p, q] \mathbf{X}[p, q]\right)^{-1} \mathbf{X}^{H}[p, q] \mathbf{Y}_{n}[p, q]
$$

In the above equation, $\mathbf{X}$ is the transmitted signal which is a matrix of $q \times p$ and $\mathbf{Y}$ is the received signal that is a matrix of $p \times q$. As mentioned in 2.1, $n$ corresponds to the receive antenna index and $q$ and $p$ are the number of subcarriers and number of symbols in each preamble, respectively.

There are two channel prediction methods used in my work to predict the channel coefficients of the preamble of the main frame. The fifth preamble is at the beginning of the main frame which is transmitted to the selected receive antenna after the training phase. The first method is to employ Lagrange Interpolation and the second one is to use Linear Regression in order to perform prediction. 


\subsubsection{Lagrange Interpolation}

In this method, the prediction is based on polynomial extrapolation which is done by means of Lagrange Interpolation and it is shown as in (2.7):

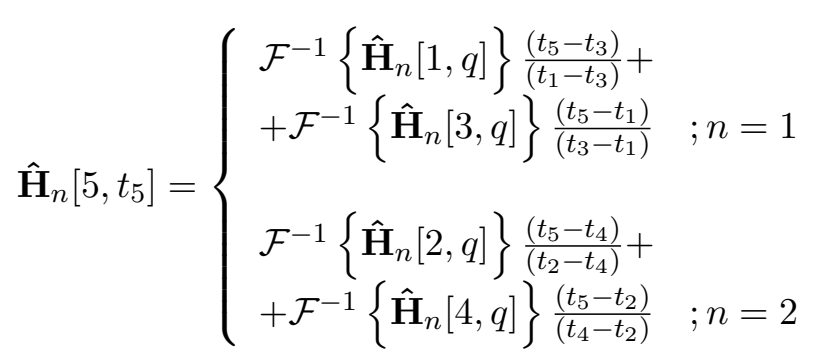

In (2.7), the times shown by $t_{i}=t_{1}, \ldots, t_{4}$ are the times regarding the transmission of the four added preambles and $\mathbf{i}$ is the time index, thus, $t_{5}$ is the time corresponding to the transmission of the fifth preamble which is the preamble at the beginning of the main frame. These times are at the end of each corresponding preamble. The channel coefficients obtained from the LS estimator are employed after inverse Fourier transform (IFT) in this equation. The estimated channel coefficients are in frequency domain, thus, by means of inverse Fourier transform, they are in time domain. The $\hat{\mathbf{H}}_{n}\left[5, \mathrm{t}_{5}\right]$ is predicted by estimated channel coefficients at the added preambles (as shown in (2.6)) and $n$ is the index of the receive antenna, since in our contribution, there are two receive antennas at the receiver side, $n=1,2$. The index $m$ which represents the number of the transmit antennas is omitted in this equation because in our scenario, there is just one antenna at the transmitter side.

At each receive antenna, the channel gain $\left|\hat{\mathbf{H}}_{n}[q]\right|^{2}$ is estimated according to the predicted channel coefficients for each subcarrier and based on these values for all subcarriers, a matrix is formed that contains the calculated values for all possible combinations of the transmit antenna and the receive antenna, which for this work is two. The matrix is the same as previously shown in Fig. 2.4. Similarly, the number of rows in this matrix is $\left(\begin{array}{l}2 \\ 1\end{array}\right)=2$ which equals the number of all switching states. Instead of calculated SNR, the matrix contains the predicted channel gain values. It has $N$ columns corresponding to the number of subcarriers. We choose the following maxmin criterion for antenna selection:

$$
\hat{n}=\arg \max _{n=1,2}\left(\min _{1 \leq q \leq N}\left|\hat{\mathbf{H}}_{n}[q]\right|^{2}\right)
$$

The minimization is over subcarriers and the maximization is over all switching states. Therefore, first in each row of this matrix, the minimum value of predicted channel gain is chosen and then a vector is formed which contains these minimum values and 
consequently the maximum channel gain value is selected in this vector which leads to the optimum receive antenna since the rows of the vector correspond to the receive antennas.

\subsubsection{Linear Regression}

The second method is to employ Linear Regression Predictor instead of Extrapolation in order to fit a predictive model for channel to a set of estimated channel coefficients of the added preambles and the corresponding time for these four preambles of the training phase. Then, the fitted model can be used to predict the channel at the beginning of the main frame, $\hat{\mathbf{H}}_{n}\left[5, t_{5}\right]$, for each $\mathrm{Rx}$ antenna. The regression polynomial is as shown in (2.9):

$$
\hat{\mathbf{H}}_{n}[p, t]=\hat{\mathbf{H}}_{0}+\hat{\mathbf{H}}_{1} t \quad ; n=1,2
$$

The curve which fits the above mentioned polynomial is obtained by Least Squares (LS) approach:

$$
\min \left|\hat{\mathbf{H}}_{n}[p, t]-\hat{\mathbf{H}}_{0}-\hat{\mathbf{H}}_{1} t\right|^{2} \quad ; n=1,2
$$

By setting the gradient to zero, the polynomial coefficients, $\hat{\mathrm{H}}_{0}$ and $\hat{\mathrm{H}}_{1}$, are found which will provide the required curve and therefore, the prediction of the channel at a later time $t_{5}$ for both receive antennas is feasible. The polynomial coefficients which are the slope and intercept of the fitting curve are as follows:

$$
\begin{array}{cc}
\hat{\mathbf{H}}_{0}=\frac{\left(\sum_{i} t_{i}{ }^{2}\right)\left(\sum_{i} \hat{\mathbf{H}}_{n}\left[p, t_{i}\right]\right)-\left(\sum_{i} t_{i}\right)\left(\sum_{i} t_{i} \hat{\mathbf{H}}_{n}\left[p, t_{i}\right]\right)}{2 \sum_{i} t_{i}{ }^{2}-\left(\sum_{i} t_{i}\right)^{2}} & ; n=1,2 \\
\hat{\mathbf{H}}_{1}=\frac{2 \sum_{i} t_{i} \hat{\mathbf{H}}_{n}\left[p, t_{i}\right]-\left(\sum_{i} t_{i}\right)\left(\sum_{i} \hat{\mathbf{H}}_{n}\left[p, t_{i}\right]\right)}{2 \sum_{i} t_{i}{ }^{2}-\left(\sum_{i} t_{i}\right)^{2}} & ; n=1,2
\end{array}
$$

Due to the transmission sequence of the added preambles to the corresponding $\mathrm{Rx}$ antennas, the values of $p$ and $\mathbf{i}$ are one and three for the first $\mathrm{Rx}$ antenna and two and four for the second $\mathrm{Rx}$ antenna. As the next step, by predicting $\hat{\mathbf{H}}_{n}\left[5, t_{5}\right]$, the same maxmin function as in (2.8) is performed for the predicted channel gain and the optimum receive antenna is selected to transmit the main frame to. 


\subsection{Performance Evaluation}

In order to evaluate the proposed antenna selection scheme, an $802.11 \mathrm{p}$ link-level simulator implemented in MATLAB is used. A carrier frequency of $f_{c}=5.9 \mathrm{GHz}$ is assumed and the system bandwidth is $B=10 \mathrm{MHz}$ as used in $802.11 \mathrm{p}$ standard. The total number of subcarriers is $N=64$ where 52 subcarriers out of total numbers are allocated for data transmission. In this scheme, Quadrature Phase Shift Keying (QPSK) and a convolutional code with code rate $R_{C}=\frac{1}{2}$ are used. In the numerical simulations, we use a time-variant channel model with an exponentially decaying power delay profile and a Clarke Doppler profile for each channel tap [31] which means that it is assumed the receive antennas are omnidirectional in azimuth. The small-scale fading processes associated with the two receive antennas are assumed to be uncorrelated.

Each OFDM frame contains 38 OFDM symbols (including the postamble symbol). For numeric Monte Carlo simulations, $F=1000$ frames are used. The resulting frame length is 200 Bytes in this simulation. The simulation is performed over two number of iterations for channel estimation. There are one transmit antenna $\left(M_{t}=1\right)$ and two receive antenna $\left(M_{r}=2\right)$. The assumed scenario is $\mathrm{V} 2 \mathrm{I}$ where the transmitter is fixed and the receiver is mobile with a velocity of $50 \mathrm{~km} / \mathrm{h} \approx 13.9 \mathrm{~m} / \mathrm{s}$. In Table 3.1, the simulation parameters which have been considered for the proposed antenna selection scheme are illustrated.

Table 2.1: Simulation Parameters

\begin{tabular}{|l|c|}
\hline Carrier Frequency $\left(f_{c}\right)$ & $5.9 \mathrm{GHz}$ \\
\hline Bandwidth $(B)$ & $10 \mathrm{MHz}$ \\
\hline Channel Model & Clarke's Doppler Profile \\
\hline Number of OFDM Symbols & 38 \\
\hline Total Number of Subcarriers & 64 \\
\hline Number of Frames & 1000 \\
\hline Frame Length & 200 Bytes \\
\hline Number of Receive Antenna & 2 \\
\hline Number of Transmit Antenna & 1 \\
\hline Velocity & $50 \mathrm{~km} / \mathrm{h}$ \\
\hline
\end{tabular}

The simulation results of the proposed antenna selection scheme is compared with the SISO case and also with the antenna selection scheme without the channel predictor in [3]. The BER results are illustrated versus $E_{\mathrm{b}} / N_{0}$, whereas $E_{\mathrm{b}}$ is the energy per bit and $N_{0}$ denotes the noise power spectral density. In (2.13), SNR calculation is shown. In this equation, $N_{\text {bpsc }}$ represents the number of coded bits per subcarrier, CR is the coding rate, $N_{\mathrm{FFT}}$ and $N_{\mathrm{G}}$ are the number of Fast Fourier Transform (FFT) points and number of samples in guard interval, respectively. $N_{\text {data }}$ is the number of data symbols in the frame and $N_{\text {pre }}$ corresponds to the number of preambles. Number of postamble 
is shown with $N_{\text {post }}$ and number of added preambles in the extended frame structure is $N_{\text {add_pre. }}$

$$
\begin{aligned}
\mathrm{SNR}= & E_{\mathrm{b}} / N_{0}+10 \log _{10}\left(\mathrm{~N}_{\mathrm{bpsc}} \cdot \mathrm{CR} \cdot\left(\mathrm{N}_{\mathrm{FFT}} /\left(\mathrm{N}_{\mathrm{G}}+\mathrm{N}_{\mathrm{FFT}}\right)\right)\right. \\
& \cdot\left(N_{\text {data }} /\left(N_{\text {data }}+N_{\text {pre }}+N_{\text {post }}+N_{\text {add_pre }}\right)\right)
\end{aligned}
$$

In order to evaluate the performance of the proposed antenna selection algorithm with the channel prediction, the results of the antenna selection with Lagrange interpolation and linear regression are compared with the results of the case in which the antenna selection is employed without any channel prediction.

First, the SIMO case with the Lagrange Interpolation predictor is compared with the SIMO case without any channel predictor in Fig. 2.5. As shown in this figure, the dashed lines are representing SIMO without channel predictor and the solid lines are corresponding to SIMO case with Lagrange Interpolation predictor. By increasing the $E_{\mathrm{b}} / N_{0}$ values from 0 to 12 , BER behavior changes gradually. Upto $8 \mathrm{~dB}$, both curves, dashed lines and solid lines are overlapping and after $8 \mathrm{~dB}$, it is inferred from this figure that diversity gain has improved remarkably when the channel prediction is used due to the steeper slope of the solid lines. Generally, it can be stated that by increasing the SNR, higher diversity gain is achievable. By looking thoroughly to the curves, it can be figured out that the slope of the solid lines are steeper than the dashed lines which results in a significant reduction in BER at an $E_{\mathrm{b}} / N_{0}$ of $12 \mathrm{~dB}$, the BER is decreased from $1.4 \cdot 10^{-3}$ to $0.5 \cdot 10^{-3}$.

In Fig. 2.6, the antenna selection scheme with Linear Regression predictor and antenna selection scheme without any channel predictor are illustrated for SIMO case. The case with the Linear Regression predictor is shown with solid lines and the case without the channel predictor is illustrated with the dashed lines. At an $E_{\mathrm{b}} / N_{0}$ of $12 \mathrm{~dB}$, the BER is decreased from $1.4 \cdot 10^{-3}$ to $0.3 \cdot 10^{-3}$ which implies that as the SNR increases the employment of the channel predictor will result in the increase of the diversity gain.

In order to evaluate the antenna selection more accurately, we also compare the results of Lagrange Interpolation predictor and Linear Regression predictor with the SISO case in which one receive antenna and one transmit antenna are used. Fig. 2.7]illustrates the comparison between their simulation results. The dashed lines are associated with two iterations of SISO case and the solid lines are related to SIMO case with antenna selection. We can show that the proposed method with both channel predictors outperforms the SISO case. The BER is improved remarkably in comparison to the SISO system by increasing the SNR values. To be more precise, it can be said that even at an $E_{\mathrm{b}} / N_{0}$ of $8 \mathrm{~dB}$, the BER is decreased from $1.4 \cdot 10^{-1}$ to $0.4 \cdot 10^{-1}$ for Lagrange Interpolation predictor and $0.3 \cdot 10^{-1}$ for Linear Regression predictor. Furthermore, at an $E_{\mathrm{b}} / N_{0}$ of $12 \mathrm{~dB}$, it is shown that the slope of the curves corresponding to the case with the 


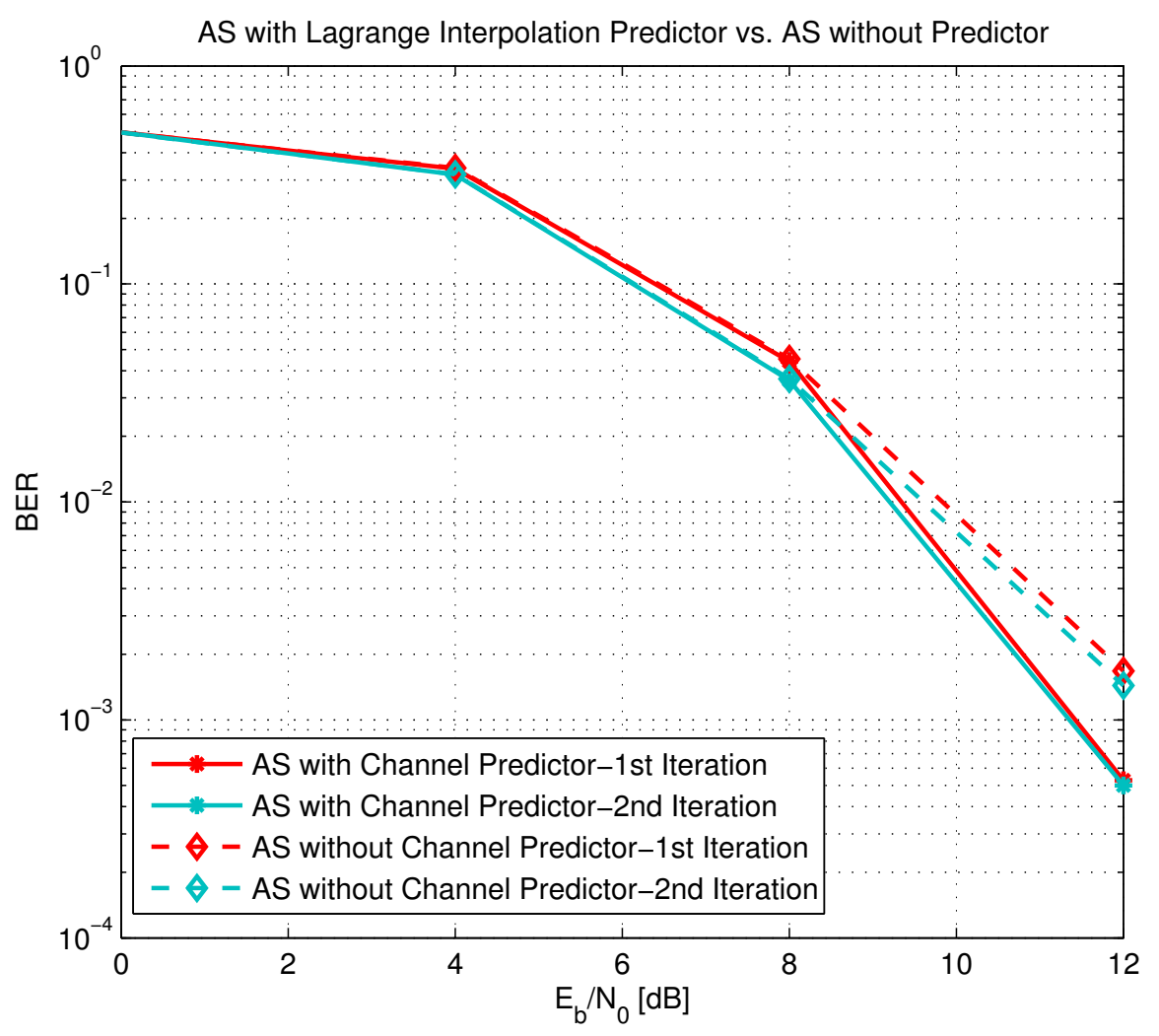

Figure 2.5: Comparison between antenna selection scheme performance with Lagrange Interpolation predictor and without any channel predictor for SIMO case (number of receive antenna $=2$; number of transmit antenna $=1$ )

channel prediction became steeper which can be inferred as remarkable improvement in diversity gain.

In Fig. 2.8, the performance evaluation of the used channel predictors, Lagrange Interpolation and Linear Regression, is shown. The solid lines correspond to Lagrange Interpolation and the dashed lines are used for Linear Regression. Both channel predictors outperform the SISO case and also the antenna selection scheme without using a channel predictor and their corresponding curves are quite close to each other especially for the $E_{\mathrm{b}} / N_{0}$ values below $8 \mathrm{~dB}$ and one channel estimation iteration. On the other hand, it can be inferred from this figure that for higher values of SNR and also by increasing the number of iterations, the slope of the curve related to Linear Regression gets steeper so that it outperforms Lagrange Interpolation. 


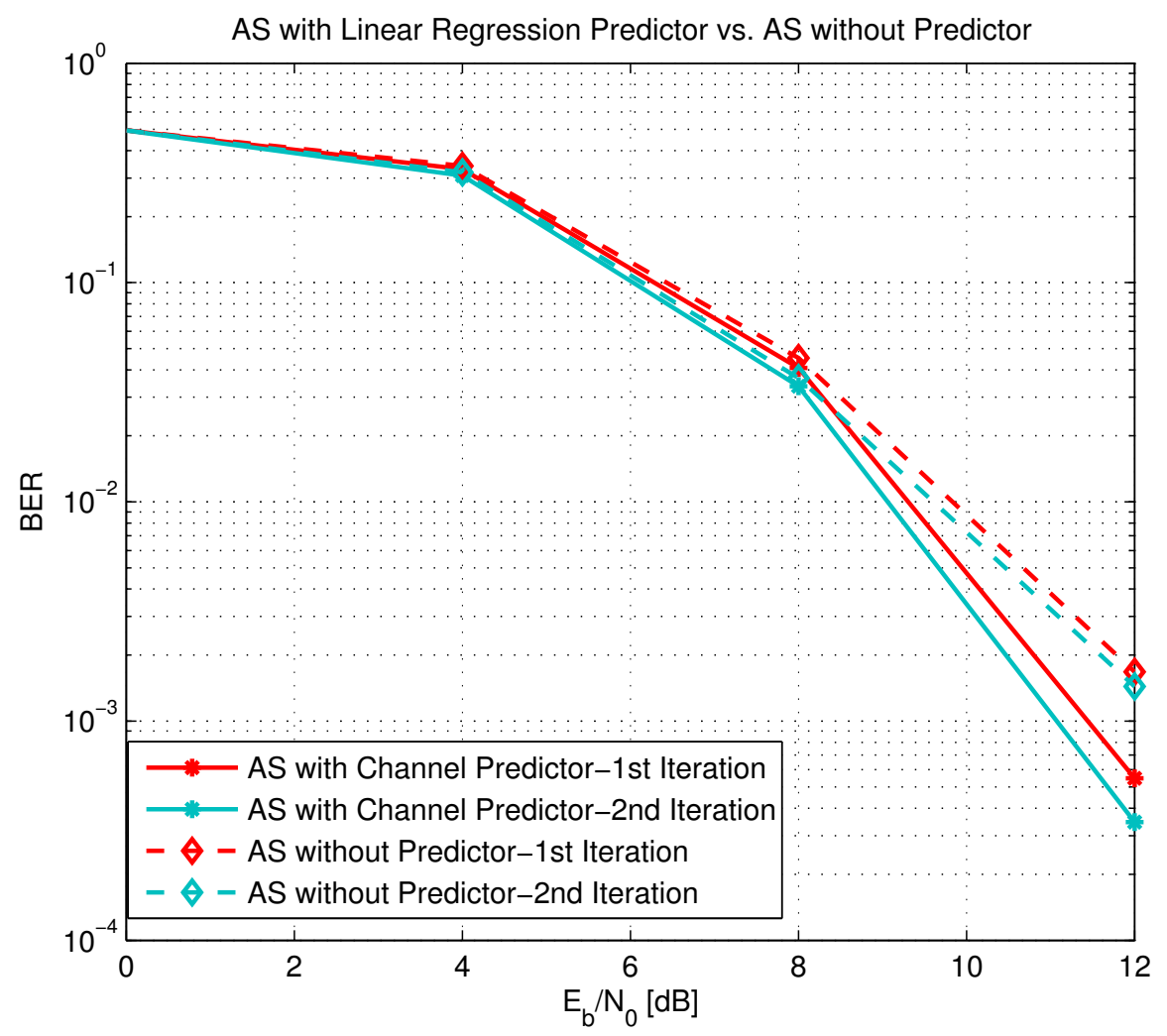

Figure 2.6: Comparative overview of antenna selection scheme performance with Linear Regression predictor and without channel predictor for SIMO case (number of receive antenna $=2$; number of transmit antenna $=1$ )

\subsection{Discussion and Conclusion}

In this chapter, I have explained the proposed antenna selection algorithm for a SIMO system with one Tx antenna and two Rx antennas. An extended version of the frame structure in IEEE 802.11p has been introduced with four additional preambles which have been used for the selection purpose. In order to perform the antenna selection, the algorithm has been presented first based on the estimated SNR and then by exploiting two different channel predictors. The employed predictors in my work are Lagrange interpolation and linear regression. The decision making process for all three methods have been described and a maxmin function has been used for this purpose which based on the simulation results can outperform some other alternatives. During this process the best link has been chosen and consequently the frame has been transmitted to the corresponding selected Rx antenna.

In order to evaluate the performance of the proposed antenna selection algorithm, Monte Carlo simulations have been done for each of the mentioned algorithms and the results 


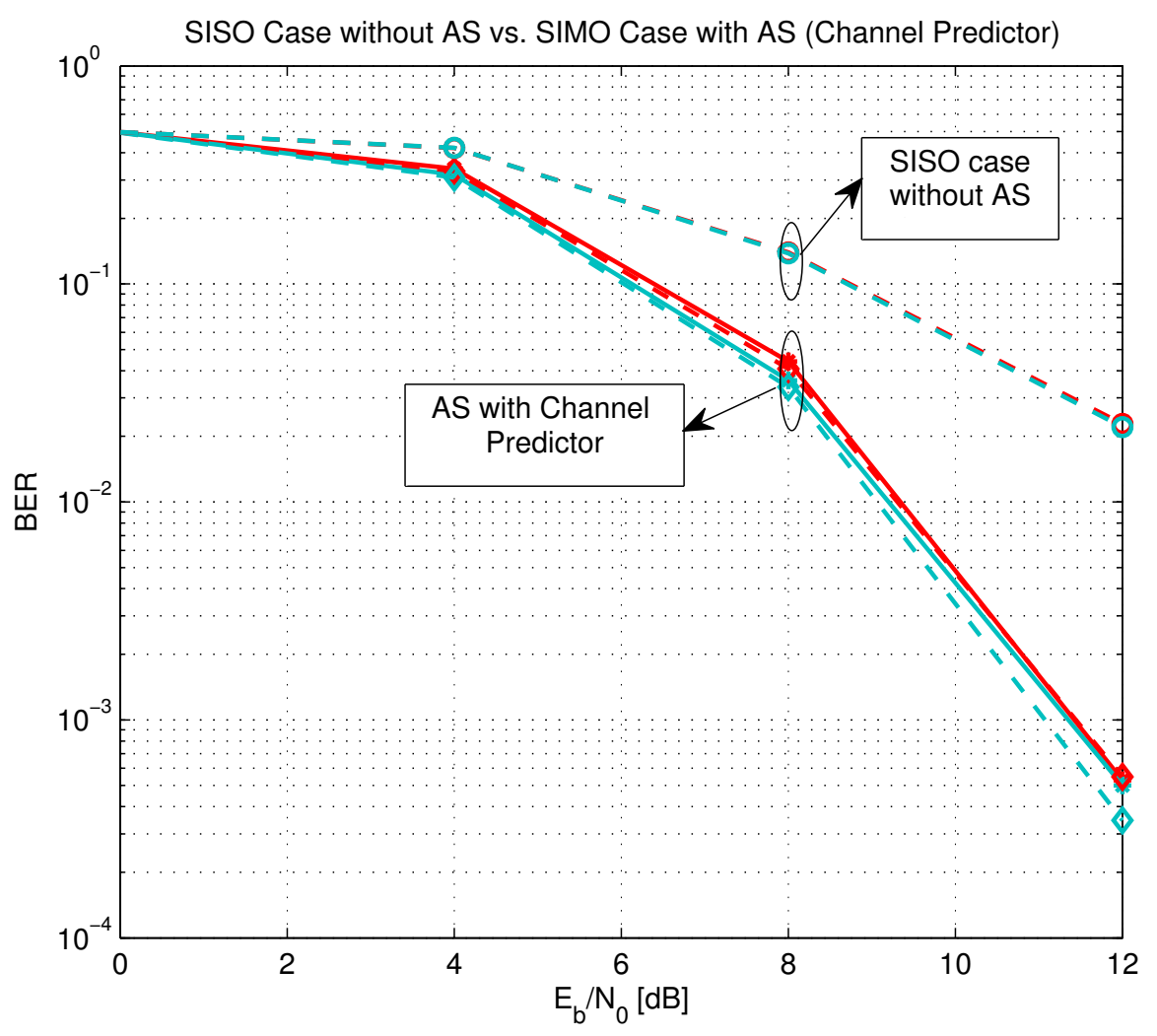

Figure 2.7: Comparative overview of antenna selection scheme performance with Lagrange Interpolation and Linear Regression predictor between SIMO case (number of receive antenna $=2$; number of transmit antenna $=1$ ) and SISO case (number of receive antenna $=1$; number of transmit antenna $=1$ ) without any antenna selection

have been compared with each other. The results have confirmed that when the channel predictors are used, higher diversity gain has been obtained comparing with the SNR-based algorithm. Moreover, the diversity gain has been improved remarkably in comparison to the SISO case. Between the used channel predictors, it has been illustrated that linear regression has outperformed Lagrange interpolation for higher values of $E_{\mathrm{b}} / N_{0}$ and also when the number of the channel estimation iterations have been increased. 


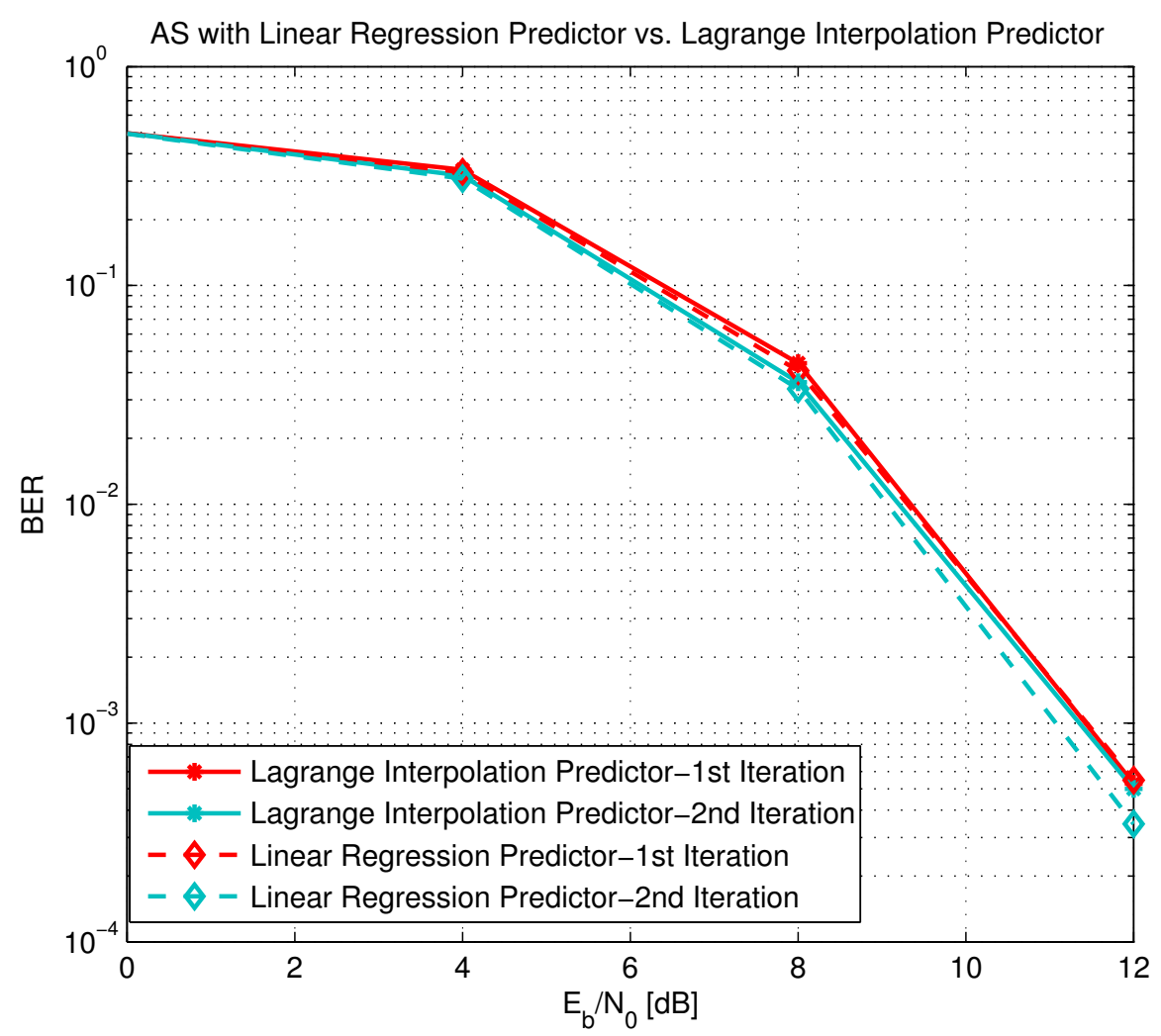

Figure 2.8: Comparative overview of antenna selection scheme performance with Linear Regression and Lagrange Interpolation predictor for SIMO case (number of receive antenna $=2$; number of transmit antenna $=1$ ) 



\section{ANTENNA SELECTION EXPERIMENT WITH SOFTWARE DEFINED RADIO DESIGN}

In this chapter, the antenna selection measurement campaign carried out in order to test the antenna selection algorithm in real environment is described in detail. The chapter is organized in a way that in the first part, LabView Communication Suite and IEEE 802.11p application framework in which the proposed antenna selection is implemented are presented. In the second part of this chapter, the measurement campaign is presented that was performed in the urban areas of Vienna, Austria including all the required equipment, measurement tracks and setup. The last section of this chapter is dedicated to the data analysis and postprocessing results obtained from the measurement.

\subsection{LabVIEW Communications System Design Suite}

LabVIEW Communications System Design Suite is a hardware design environment in which the communications systems can be prototyped [32]. There are two application frameworks to implement standard protocols; Long-Term Evolution (LTE) and IEEE 802.11 application frameworks. In the latter application framework, a real-time OFDM PHY and lower Media Access Control (MAC) are provided for implementation of any IEEE 802.11-based system. This design suite provides all the required tools to create an application, integrate it into your system in order to be used with hardware and deploy the application to a Field-programmable Gate Array (FPGA). Since in this work, the main focus is on the antenna selection diversity in IEEE 802.11p, the second application framework was used to implement both the required modifications for IEEE 802.11p and the proposed antenna selection algorithm.

\subsubsection{IEEE 802.11 Application Framework}

Since my work is based on IEEE 802.11p standard, IEEE 802.11 application framework is used to evaluate the proposed antenna selection algorithm. In this application framework, the PHY and MAC layers are implemented in modular blocks by means of 
LabView Communications and it can be simply modified based on the standard. With such framework, there is the possibility to examine new algorithms and multi-antenna architectures. Basically, the application framework supports IEEE 802.11a and 802.11ac standards. This framework is compliant with IEEE 802.11 standard, thus, to use this application framework for implementing the algorithm in IEEE 802.11p, several modifications have been implemented which are specified in this part.

The functionalities which are provided by the PHY transmitter are scrambler, convolutional coding and bit interleaving, binary Phase-shift Keying/Quadrature Amplitude Modulation (BPSK/QAM) constellation mapper up to 256-QAM, pilot sequence generation, signal fields generation, OFDM symbol generation based on Inverse Fast Fourier Transform (IFFT), guard interval (GI) insertion and addition of training fields.

Corresponding functionalities provided for the PHY receiver side are clear Channel Assessment (CCA) based on energy detection as well as on signal detection, packet detection, Automatic Gain Control (AGC), time and frequency synchronization, GI removal, OFDM symbol demodulation based on FFT, channel estimation and zero-forcing equalization, BPSK/QAM demmapping, deinterleaving, convolutional decoding using Viterbi decoding, descrambling and signal fields decoding and reconfiguring the receiver based on signal fields information.

The functionalities listed above are mapped to a host processor, target FPGA and RF hardware. The architectural split is shown in the Fig. 3.1. Lower MAC and baseband PHY layer algorithms are included in FPGA target for the transmitter and receiver. The host processor is assigned to upper MAC layer functions such as protocol control. As it is also shown in this figure, a message-based Interface Communication Protocol (ICP) is used for the data path interface between the host and the target.

The IEEE 802.11 application framework is a standard compliant framework, thus, the required modifications have been done in order to implement IEEE $802.11 \mathrm{p}$ in this application framework. There are two major required modifications. The first modification is regarding the bandwidth which is $20 \mathrm{MHz}$ in the application framework and for IEEE $802.11 \mathrm{p}$, it should be $10 \mathrm{MHz}$. Generally, all OFDM timing parameters are doubled in value. In other words, the data clock cycles per OFDM symbol has been changed to 960 cycles instead of 480 cycles and consequently, the OFDM symbol duration will change from $4 \mu \mathrm{s}$ to $8 \mu \mathrm{s}$ as depicted in Fig. 3.2 with red rectangle. In addition to the symbol duration, the sampling rate has been modified at the receiver and the transmitter. Thus, the default value which is $80 \mathrm{MS} / \mathrm{s}$ is now reduced to $40 \mathrm{MS} / \mathrm{s}$. These changes have been applied at the host side as shown in Fig. 3.3 and Fig. 3.4. At the transmitter side, this is the sampling rate obtained from the transmitter chain and at the receiver side, this sample rate is fed to the receiver chain. The implemented modifications are shown with red rectangles in these figures.

Since the main point of this measurement campaign was to test the performance of the antenna selection algorithm, the requirements have been added and implemented 


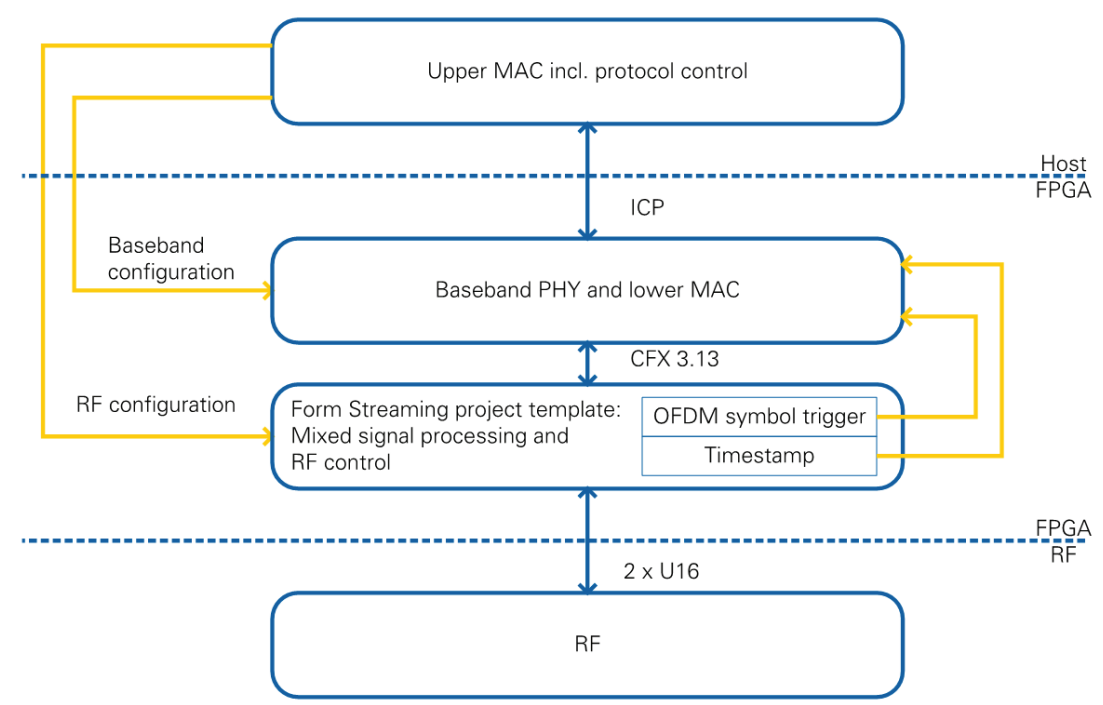

Figure 3.1: Functional split between Host, FPGA and RF

in the application framework. So the second part of the modifications is assigned to the adaptations required for the antenna selection algorithm. The adaptations include first the transmission of multiple preambles and reception of multiple preambles for the purpose of antenna selection. Then, the power of the received signal for each $\mathrm{Rx}$ antenna is calculated as in (2.4). This power is corresponding to the two added preambles at each receive antenna. The maxmin function is implemented at the receiver side for the decision making process for the receive antenna selection. The metric which was needed to evaluate the performance of the proposed algorithm is PER that has been also defined in the application framework.

In Fig. 3.5, the whole process is shown as a diagram including the four added preambles and the parts related to the received signal power calculation. By following the flow of the diagram, it is observed that two different blocks estimate the received minimum power of the received signals for both $\mathrm{Rx}$ antennas and then maximum value is chosen to select the Rx antenna according to (2.5) .

\subsection{Antenna Selection Diversity Measurement Campaign}

The measurement campaign was done in Vienna urban areas in Austria for V2I communications and three different locations were chosen to test the antenna selection algorithm 


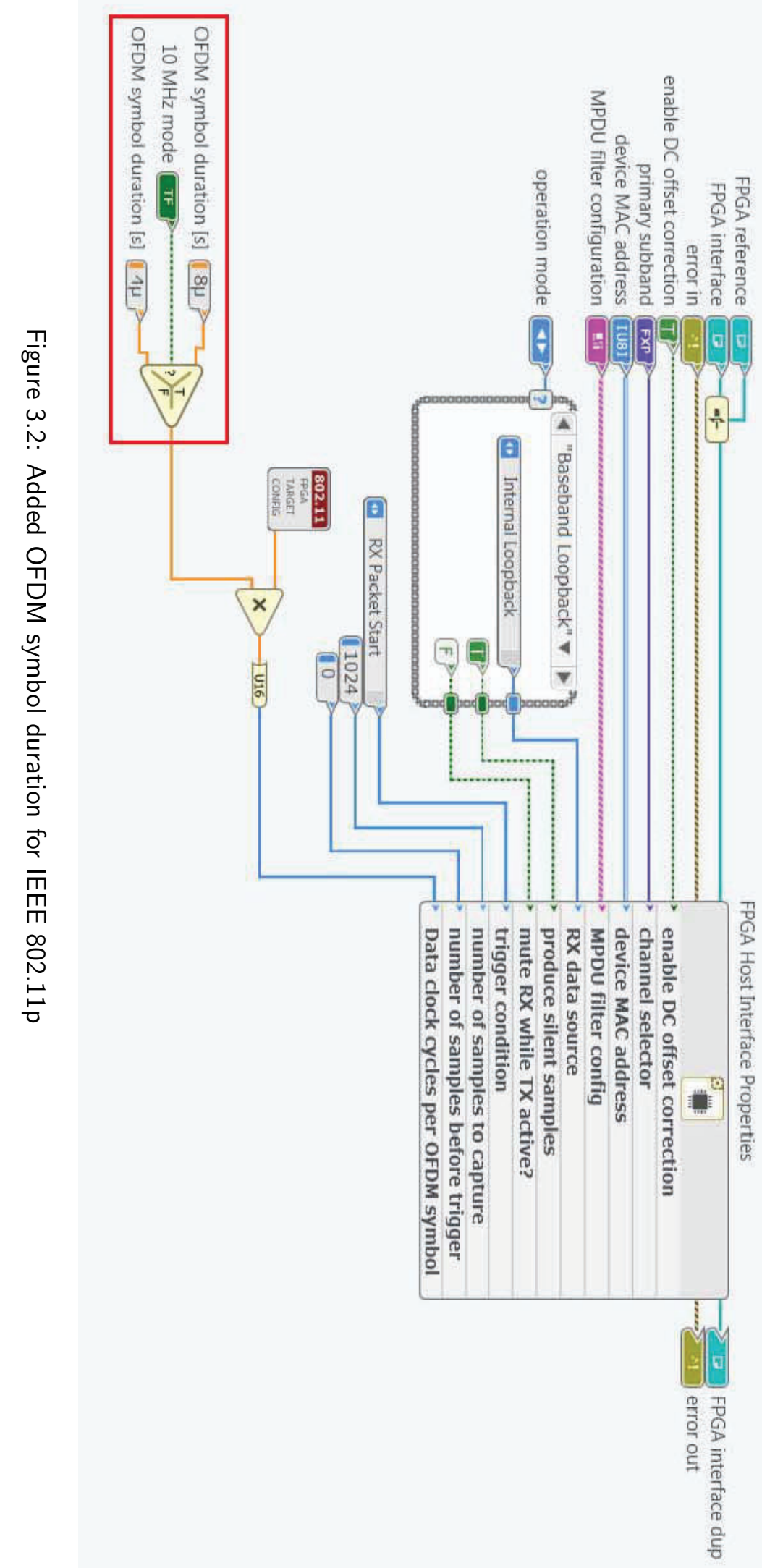




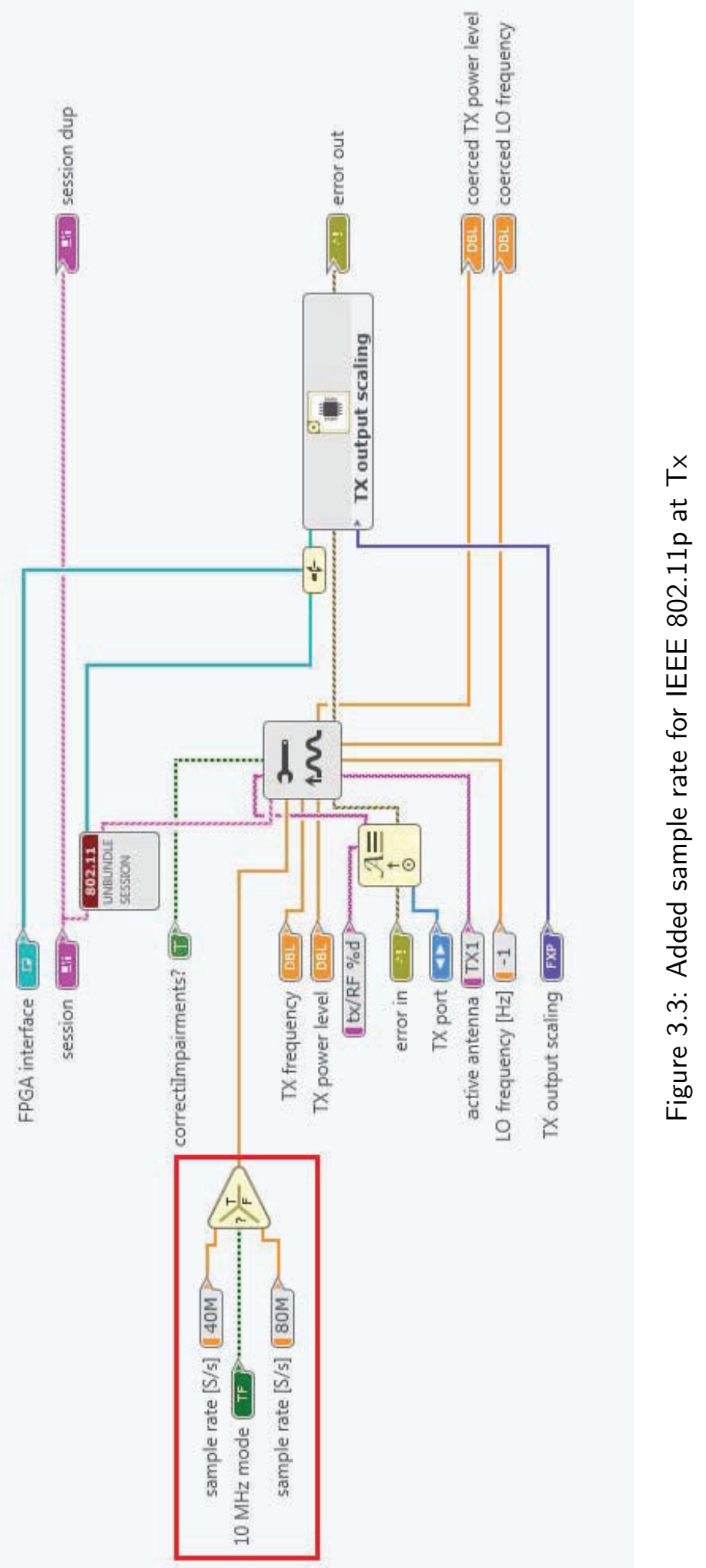




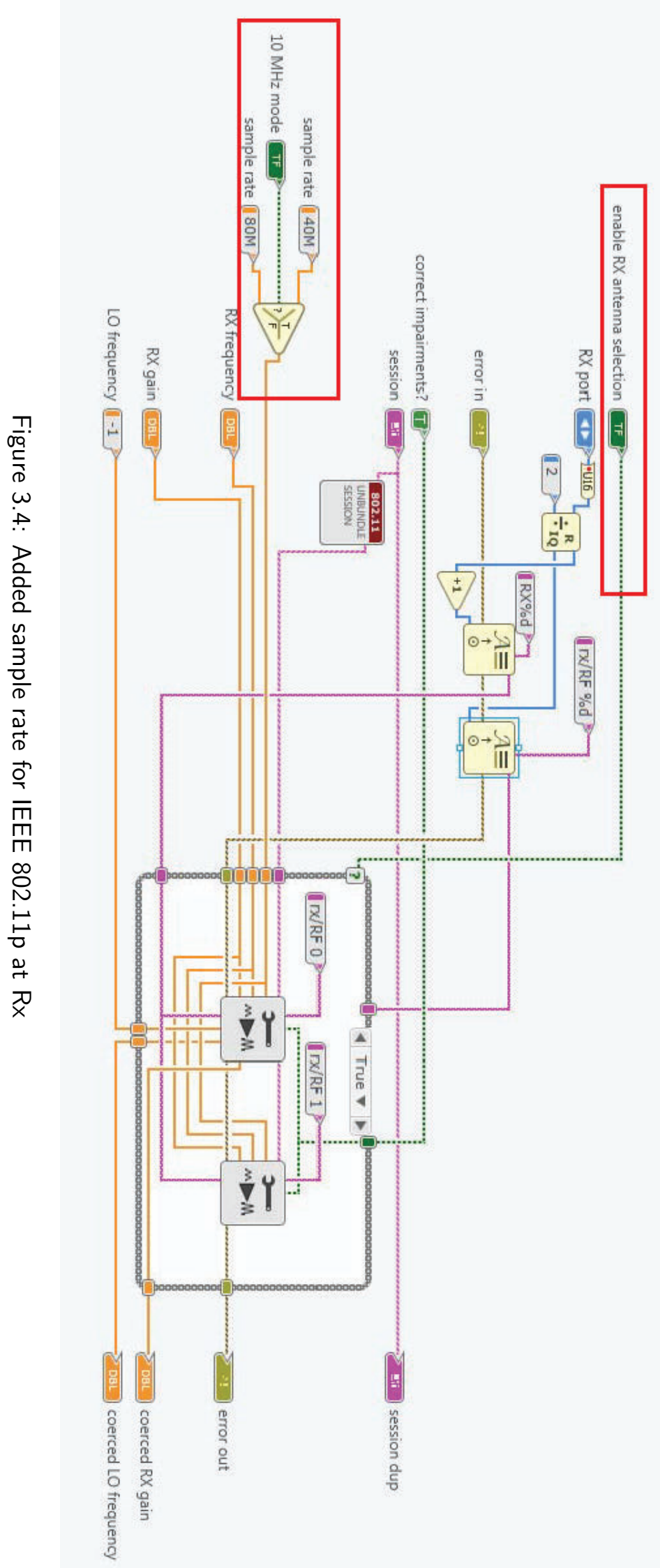




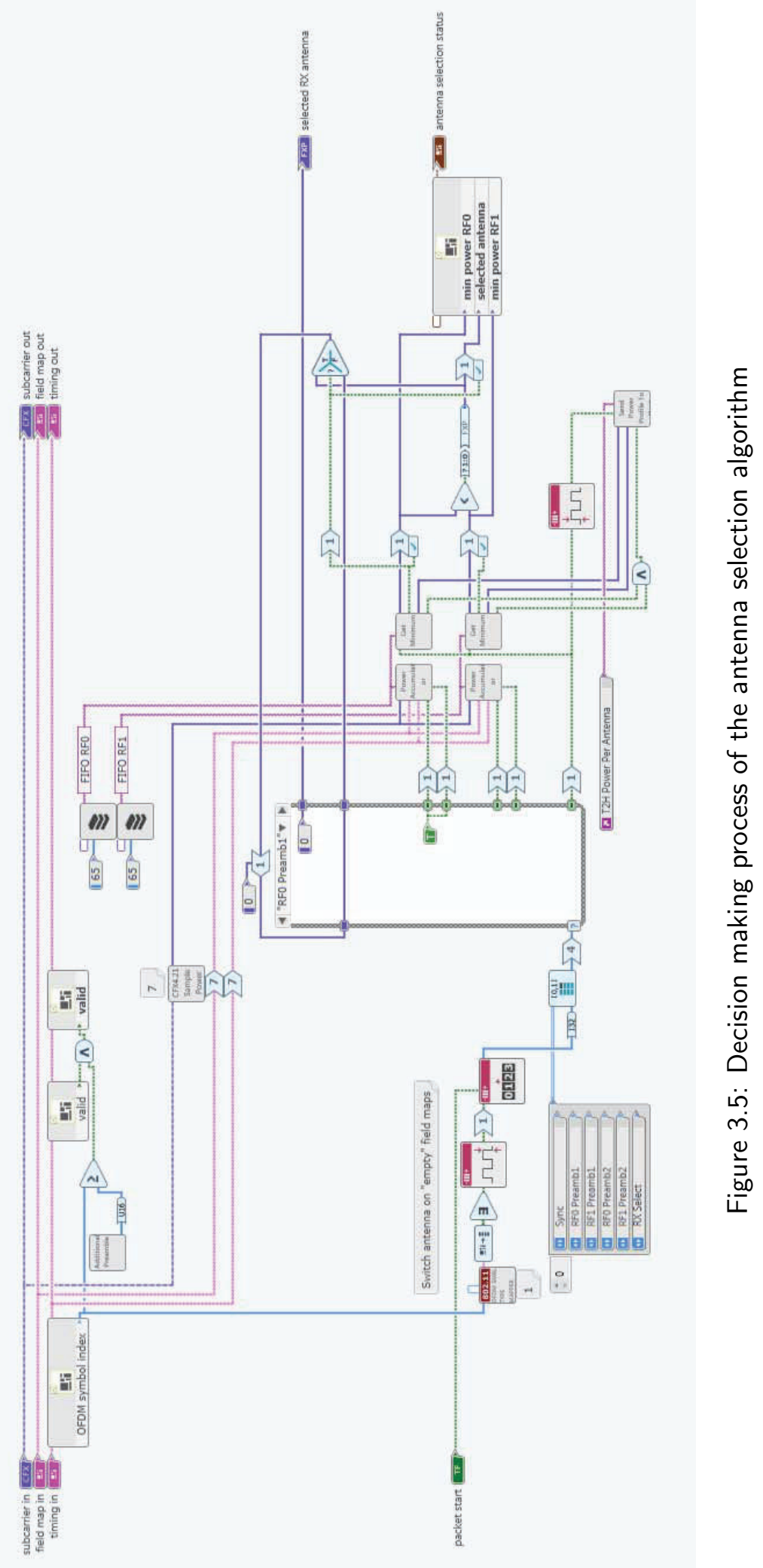


performance in real environment. The first location features a direct path where the transmit ( $T x)$ antenna was located somehow in the middle of the measurement track as shown in Fig. 3.6 and the distance driven by the car is approximately $300 \mathrm{~m}$. The second location is an L-Turn which is a one way street with an intersection and the transmit antenna is mounted close to the corner of the street as in Fig. 3.7. The vehicle is driven on the one way street and it turns left at the intersection. The driving distance for this scenario is about $200 \mathrm{~m}$. The last one is a direct path under the subway station. The measurement track is surrounded from one side with tall buildings and from the other side with the bridge of the subway train rail as shown in Fig. 3.8. In all of the figures, the exact position of the Tx antenna is marked with a red dot based on Global Positioning System (GPS) data; in Table 3.1, the exact position of Tx is listed for all three scenarios with the latitude and longitude. The measurement track is also shown with an orange line in all figures.

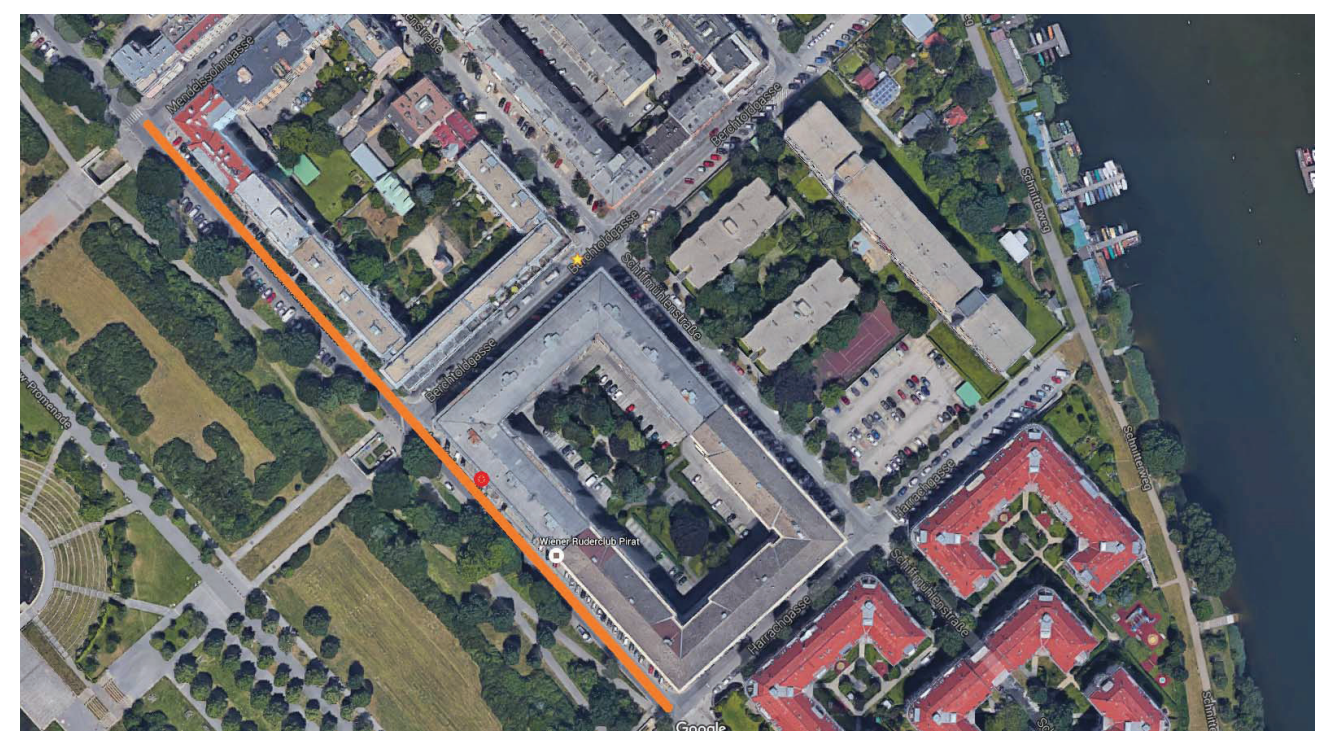

Figure 3.6: Measurement track for direct path

Table 3.1: Transmit antenna location for the measurement campaign for each scenario

\begin{tabular}{|l|c|c|}
\hline & Latitude (N) & Longitude (E) \\
\hline Direct Path & 48.223085 & 16.424222 \\
\hline L-Turn & 48.223806 & 16.424694 \\
\hline Subway Vicinity & 48.237056 & 16.422917 \\
\hline
\end{tabular}




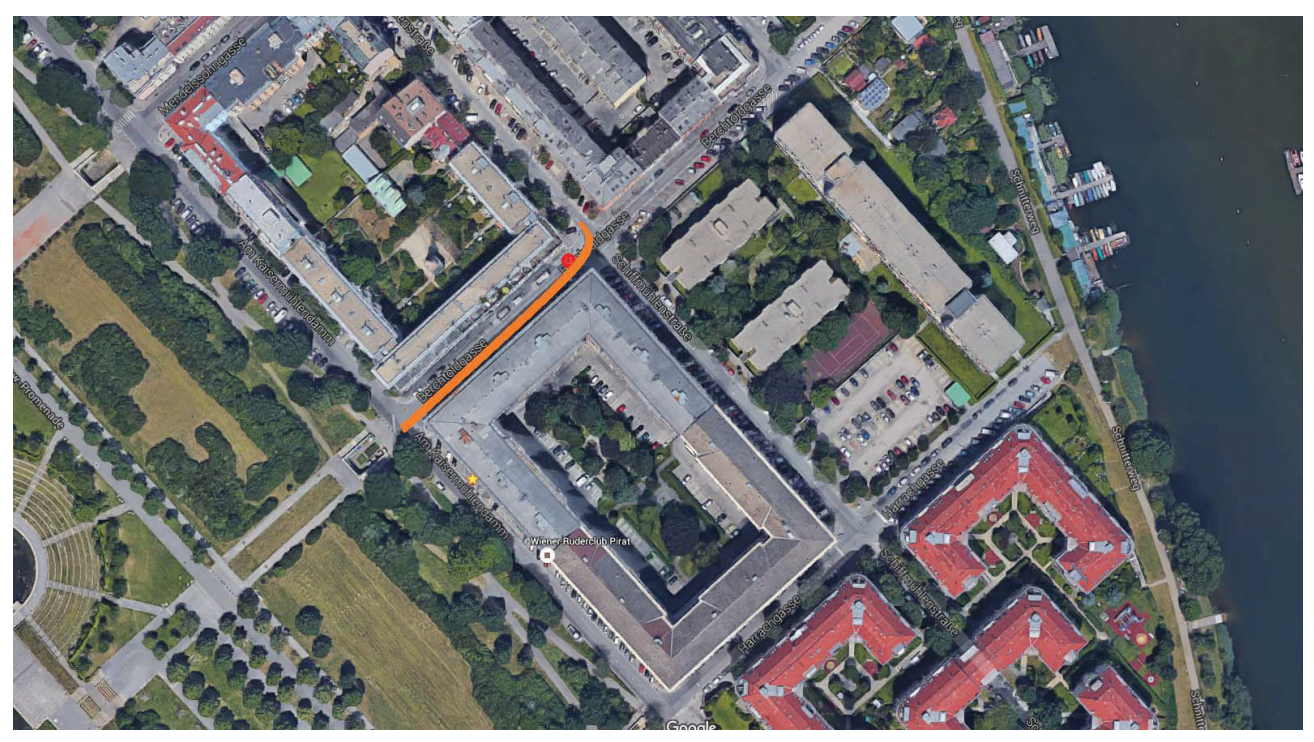

Figure 3.7: Measurement track for L-Turn

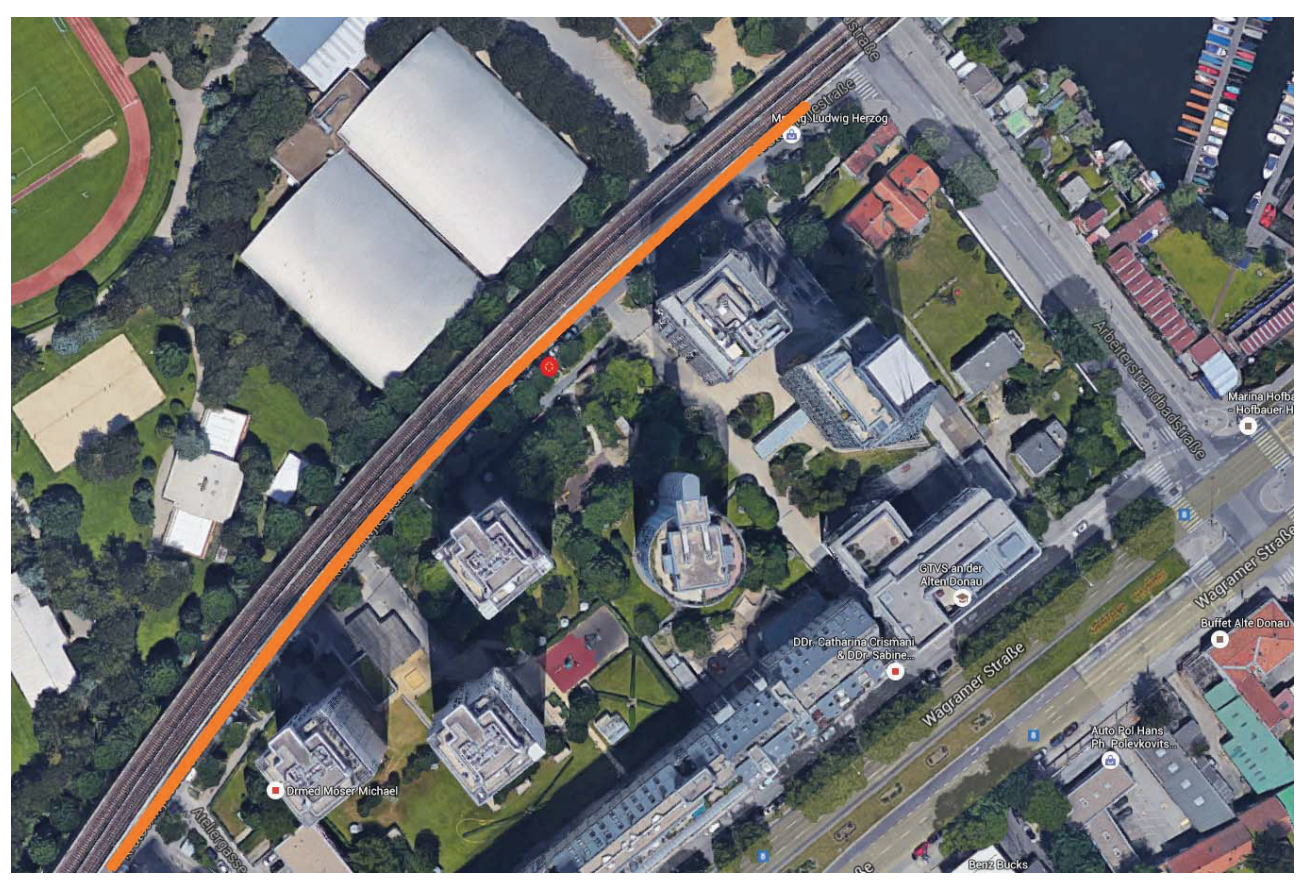

Figure 3.8: Measurement track for Under Subway Path 


\subsubsection{Measurement Setup}

The measurement for $\mathrm{V} 2 \mathrm{I}$ scenario was done for three different scenarios with one $\mathrm{Tx}$ antenna at a fixed position as RSU and two Rx antennas mounted on the roof of the vehicle. In this section, I will explain the measurement setup and the equipment which were used in this campaign.

In this measurement, the antenna type used at the transmitter side is different from the ones used at the receiver side. Therefore, antenna parameters are summarized for both types of antenna in Table 3.2 and in the Fig. 3.9 and Fig. 3.11 the antennas are shown. As it is shown in Fig. 3.11, the two receive antennas mounted on the roof of the vehicle has a $90{ }^{\circ}$ angle with each other. As receive antenna, two cooperative vehicleinfrastructure systems are used with magnet feet. At the transmitter side, vertically polarized omnidirectional antenna was used.

Table 3.2: Physical parameters for the transmitter and receiver antennas

\begin{tabular}{|l|c|c|}
\hline Parameter & Tx Antenna & Rx Antenna \\
\hline Type & omni-directional & directional \\
\hline Gain $[\mathrm{dBi}]$ & 9 & 14 \\
\hline Center Frequency $[\mathrm{GHz}]$ & 5.9 & 5.9 \\
\hline Transmit Power $[\mathrm{dBm}]$ & 9 & - \\
\hline EIRP $[\mathrm{dBm}]$ & 18 & - \\
\hline $3 \mathrm{~dB}$ Beamwidth hor. $/$ ver. $\left[^{\circ}\right]$ & $360 / 14$ & $40 / 30$ \\
\hline Polarization & vertical & vertical \\
\hline Height $[\mathrm{m}]$ & 1.6 & 2 \\
\hline
\end{tabular}

The measurement parameters are listed in Table 3.3. During the measurement, the speed of the vehicle was almost between 20 and $30 \mathrm{~km} / \mathrm{h}(5.5$ and $8.3 \mathrm{~m} / \mathrm{s})$. The length of the packet was set too 200 Bytes which is the maximum packet size allowed for CVIS transmit antenna.

Table 3.3: Measurement parameters for the antenna selection measurement campaign

\begin{tabular}{|l|c|}
\hline Parameter & Value \\
\hline Packet length [Byte] & 200 \\
\hline Transmit rate [packets/s] & 1000 \\
\hline MCS & QPSK $1 / 2$ \\
\hline Average velocity $[\mathrm{km} / \mathrm{h}]$ & 20 \\
\hline
\end{tabular}

In this measurement, the NI USRP-2953R, a Software Defined Radio (SDR) platform, was used at the receiver and transmitter sides. Since Universal Software Radio Peripheral (USRP) RIO is a $2 \times 2$ MIMO transceiver, one was used at the receiver side with two 


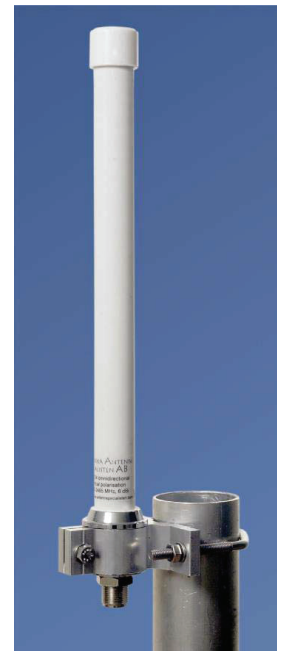

Figure 3.9: Omnidirectional Antenna as $T x$

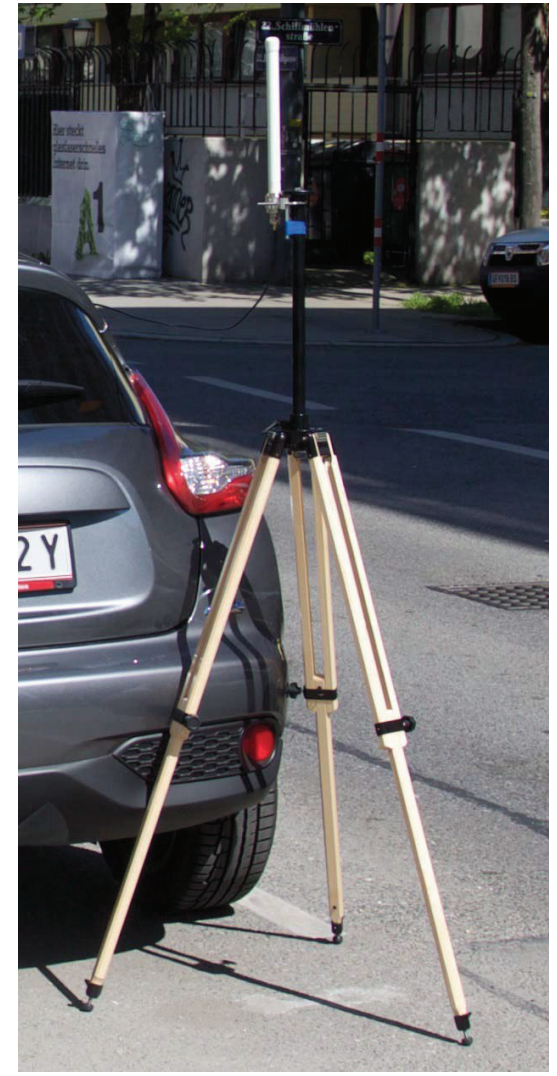

Figure 3.10: Antenna mounted at a fixed position as $T x$ 


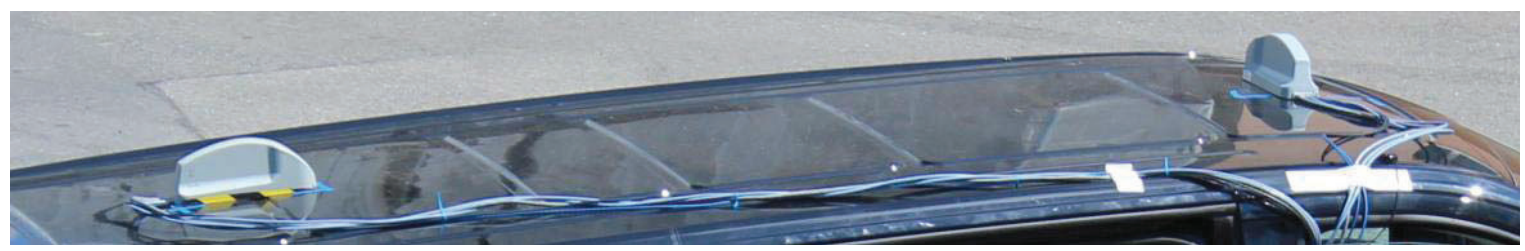

Figure 3.11: Antennas mounted on the roof of the vehicle as Rx

receive antennas connected to the ports and one was used at the transmitter side for the transmit antenna. Fig. 3.12 shows the exact USRP RIO model that was used in this measurement campaign.

In addition to USRPs, at both receiver and transmitter sides, there was PXI chassis, a PC-based platform for measurement and automation systems [7]. PXI is a highperformance platform for applications like automotive and industrial test. In Fig. 3.13, PXle-1082 which has been used in the measurement campaign is shown. This PXI is an 8-slot chassis with up to $8 \mathrm{~GB} / \mathrm{s}$ system bandwidth.

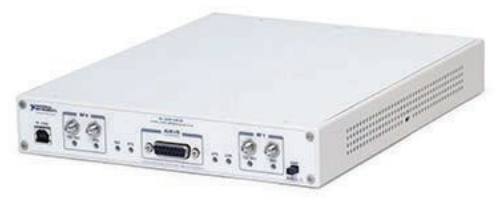

Figure 3.12: USRP-2953R [6]

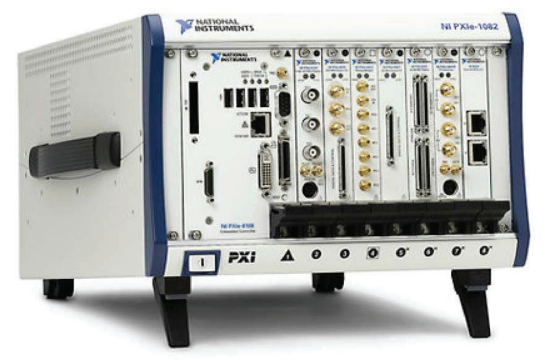

Figure 3.13: PXle-1082 (PXI Express chassis) [7]

In this measurement campaign, gel batteries are used as power supply at both receiver and transmitter sides which has Valve Regulated Lead Acid (VRLA) technology. This technology provides sealing for the battery so that in case of any overcharging or cell failure, the gas can be depleted through a valve. Such batteries in which the electrolyte is immobilized as gel have longer service life and better cycle capacity than AGM (Absorbent Glass Mat) batteries.

In addition to the above mentioned batteries, Multiplus Compact DC/AC inverter is used during the measurement. This inverter has two AC outputs and is Lithium lon battery compatible.

\subsubsection{Data Analysis and Performance Evaluation}

In this section, the data analysis results from the antenna selection measurement campaign are illustrated and discussed. In this part, the results are shown for three different 
scenarios and for each one, antenna selection algorithm and also SISO case have been tested separately to evaluate the performance of the proposed algorithm. In the first case, where antenna selection is employed, 12 rounds of measurement were performed for each scenario and for the SISO case, 12 rounds were done as well. As mentioned before, the Rx antennas had an angle of $90{ }^{\circ}$ on the roof of the vehicle. Thus, the 12 rounds of the measurement for the SISO case were divided into two sets of rounds. In the first 6 rounds, the rear antenna was connected and acted as Rx antenna and for the second 6 rounds, the front antenna was chosen as Rx.

GPS logged data, measured PER values and the video for each round of the measurement were captured and collected during the antenna selection measurement campaign to be used for the performance evaluation. It should be noted that for a more accurate data analysis, the captured GPS data was used to obtain the driven distance per second for each round of scenarios. A GPS receiver module was connected to a laptop to capture GPS data. The GPS data was logged per second and captured separately for each round of the measurement. A camera was also provided at the receiver side to record each round of the measurement and to be used for the further data processing.

As mentioned before, one of the implemented modifications in the application framework for my work was to define PER. PER estimation is formulated as in (3.1). The correctly received packets are depicted by $N_{r p}$ with a 32 bit Cyclic Redundancy Check (CRC) and the total number of transmitted packets are shown with $N_{t p}$ and the estimated PER values are averaged every $250 \mathrm{~ms}$.

$$
\widehat{\mathrm{PER}}=1-\frac{\widehat{N}_{r p}}{N_{t p}}
$$

Due to the bad resolution of the GPS device, it happened that for some rounds, the logged GPS data contained mostly the outliers and the whole round was excluded. Additionally, in some rounds, the PER values were not captured in a right way. Based on the mentioned issues, in the shown results, the number of rounds are not equal to the number of total rounds done for each scenario, which means that the number of curves plotted based on the captured data is not necessarily equal to the total number of measurement rounds. The rounds in which the captured data and the logged GPS data are both valid and could be used for postprocessing are named "valid rounds" from now on in this thesis.

In the next subsections, each scenario is described separately and there will be also the comparison between the antenna selection algorithm with the SISO case with one $\mathrm{Rx}$ and one Tx antenna. The results are shown by means of PER values and the distance. To calculate the distance, first, it was required to know the start time and the end time of each measurement round which was obtained from the video records and also the screen-shots of the application framework's panel. Since in each set of captured 
PER values, each value was an average over $250 \mathrm{~ms}$, the total duration of each round could be also used to be more precise. Then, the GPS data was used to calculate the driven distance per second and therefore, to obtain the distance between PER values, interpolation was used.

In each scenario, the performance evaluation results are shown in two figure for each case; the antenna selection and the SISO case. The difference of these figures are in the horizontal axis which corresponds to the distance. In one of the figures, the horizontal axis depicts the total driven distance which was calculated as explained previously. In the next one, since the minimum value of PER in at the RSU or in a very close vicinity of it, the exact location of the RSU, based on the latitude and longitude, has been considered as the central point which all minimum PER values are happening and the distance between the RSU location and each point of PER values are calculated. So, the main difference between these figures are the horizontal axis and the overlapping curves.

\section{Direct Path Scenario}

In the first scenario which has been already explained in section 3.2, the vehicle is driven along a direct path in which the distance between the start and stop points was $300 \mathrm{~m}$ and the transmit antenna is mounted at $180 \mathrm{~m}$ from the starting point of the measurement track.

In Fig. 3.14 PER performance evaluation of SISO case is shown while the vehicle drives along a direct path. The vertical axis corresponds to PER values and the horizontal axis corresponds to the total driven distance. Here, the total number of measurement rounds that the GPS data and PER values were both valid is eight. In the first four rounds, the rear antenna was chosen at the receiver side which in this figure are shown with solid lines. For the second four rounds, the front antenna is the receive antenna and the curves with cross marks shown in this figure corresponds to these measurement rounds. The PER values start at 1 which is the highest value and as the vehicle moves towards the RSU, it starts to decrease, then, by approaching the RSU, this value reaches its lowest point and after passing the RSU, it starts to get increased again. The results show that PER values reach lower values when the front antenna is chosen as the receive antenna than the rounds in which the rear antenna was the Rx antenna in this scenario. The lowest PER value for the front antenna is 0.22 which corresponds to round five of the measurement while this value for the rear antenna is 0.34 for the third round of the measurement.

As illustrated in Fig. 3.14 on the horizontal axis the total driven distance is shown and since the duration of each measurement round was different due to the delay of the framework and the equipment, consequently, the curves are not overlapped and also the total distance for each round is different from the other. Therefore, in Fig. 3.15, 


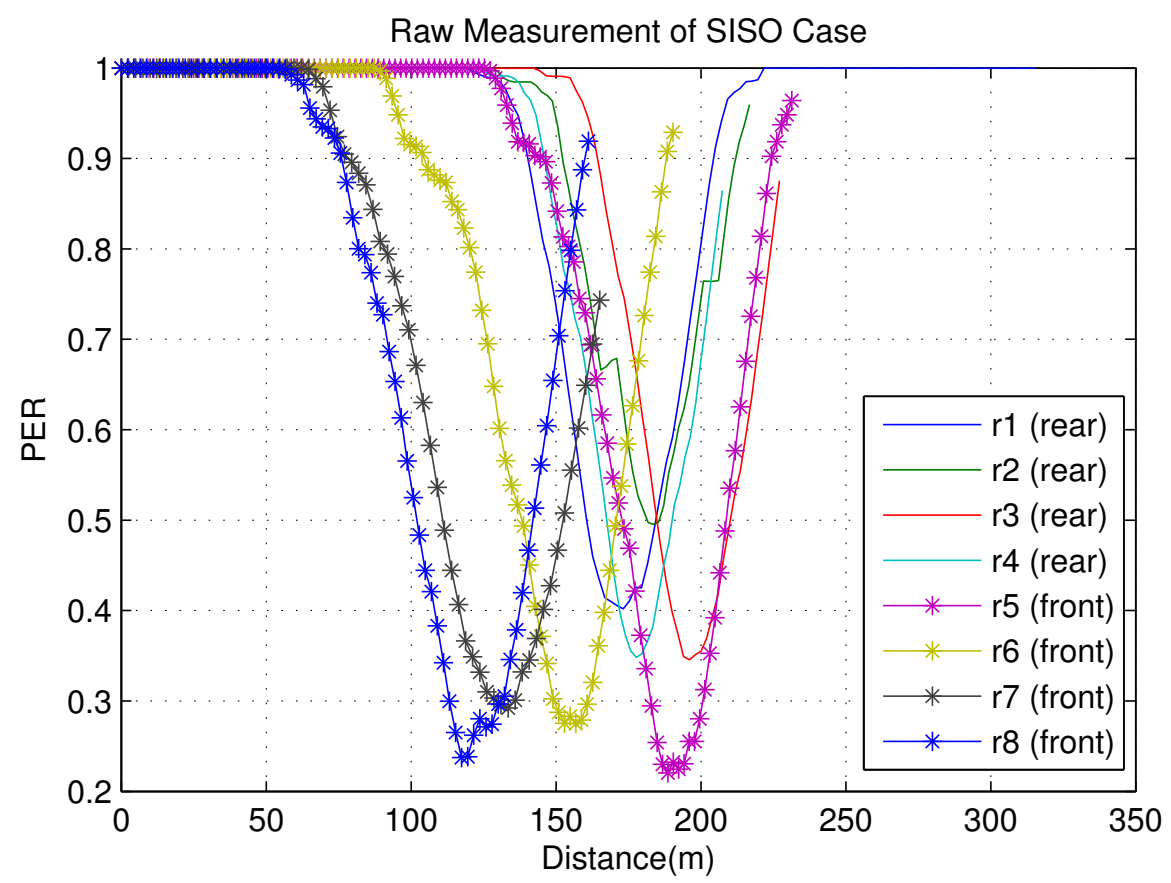

Figure 3.14: Performance evaluation of SISO case in direct path scenario - PER vs. total driven distance

the PER values are shown based on the exact location of the RSU and the curves are overlapped as well. In this figure, when all the curves are overlapped, the difference between choosing the rear and the front antennas as Rx antenna are clearly shown in the minimum point of the curves.

After testing the SISO case, the antenna selection algorithm was tested for the direct path scenario. There are seven valid rounds of measurements out of total 12 rounds. As mentioned above, the results are shown as PER vs. distance in two different ways; PER vs. total driven distance (as in Fig. 3.16) and PER vs. distance from RSU (as in Fig. 3.17). The lowest PER value in this case is 0.05 which is for the first round of this scenario. Comparing to lowest value of PER in the SISO case, it confirms that in such scenario, antenna selection algorithm outperforms the case in which just one single antenna is at the receiver side. In the case where antenna selection is employed, the minimum values of PER lie between 0.05 and 0.2 . While for the SISO case, this range is 0.2 to 0.5 . In Fig. 3.17, the behavior of the overlapped curves shows that the measurement rounds were very repeatable.

\section{L-Turn Scenario}

For the second scenario, the receiver was driven in a street which the RSU was located so close to a crossing on this street. The exact measurement track has been already shown 


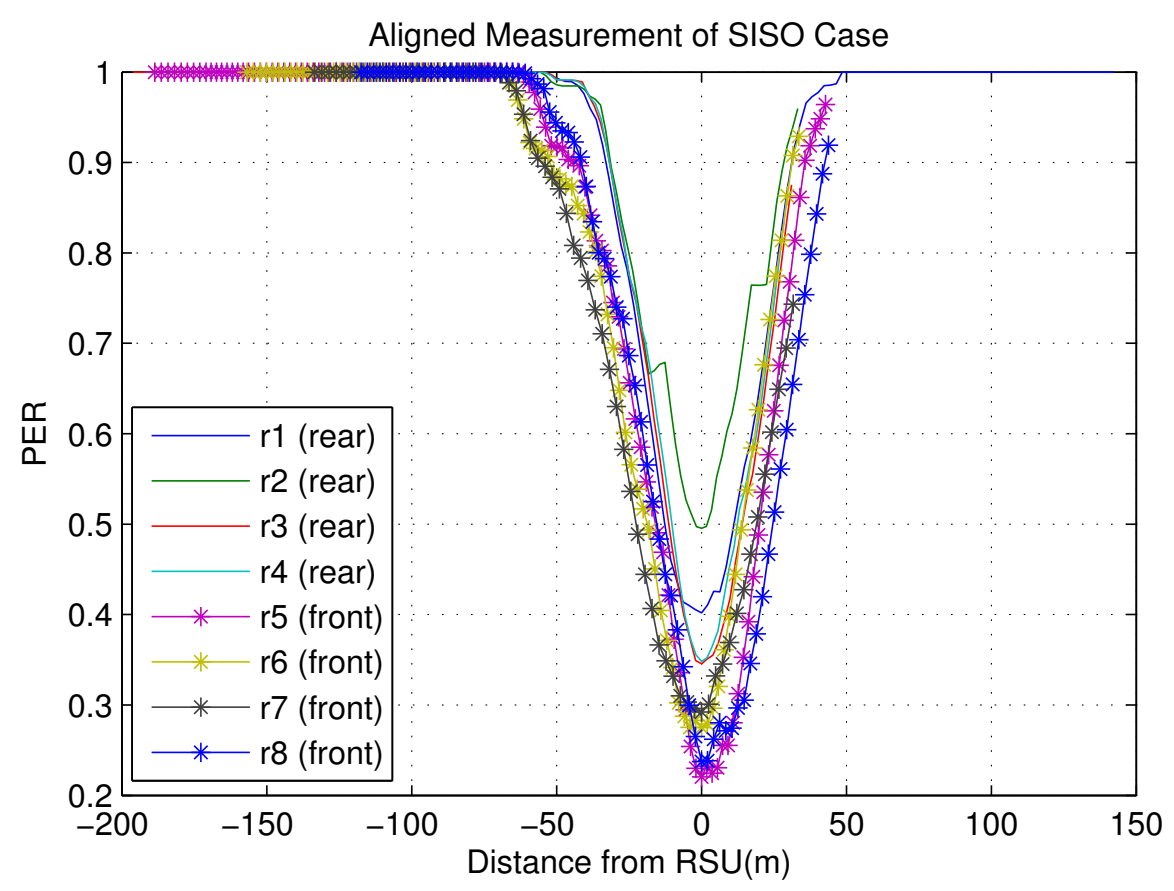

Figure 3.15: Performance evaluation of SISO case in direct path scenario - PER vs. distance from RSU

in Fig. 3.7. The whole distance is about $130 \mathrm{~m}$ and the RSU is almost at the end of the driving path. First, the SISO case results are illustrated and then, the performance evaluation results for the antenna selection algorithm.

In this measurement scenario, the SISO case has been tested and as already mentioned in the direct path scenario, the total number of measurement rounds is divided into two and in the first set of rounds, the rear antenna was the Rx antenna and in the second set of measurement rounds, the front antenna was chosen as $R x$ antenna.

In the first figure, Fig. 3.18, the PER values are shown vs. the total driven distance for the SISO case. As it is shown, the captured data is for 8 valid rounds of measurement, since from the remaining rounds, valid data was not obtained. In this figure, the first 4 rounds of the measurement with marked lines correspond to the rear antenna acting as $\mathrm{Rx}$ antenna. The next four rounds are the data for the front antenna.

The next figure shows the results of PER versus the distance of the Rx antenna from the RSU. In Fig. 3.19, the lines with the marks are for the rounds in which the front antenna was the Rx antenna. Among the measurement rounds for the rear antenna, the minimum PER value is 0.006 for the 5 th round while for the front antenna, this value at its lowest point can reach 0.003 in the 3 rd round of measurement.

In the second part of the measurement in the L-Turn scenario, the performance of the antenna selection algorithm has been investigated and the results are shown in Fig. 3.20 


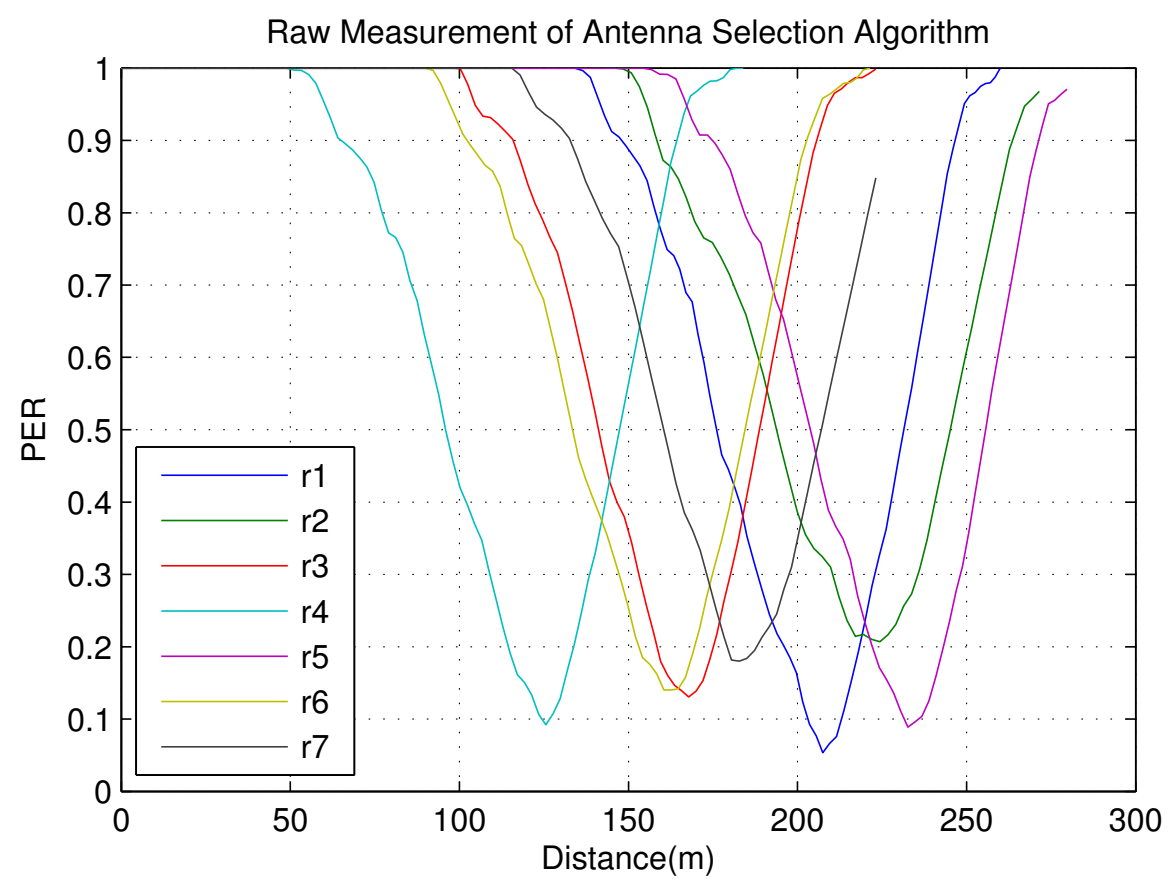

Figure 3.16: Performance evaluation of antenna selection algorithm in direct path scenario - PER vs. total driven distance

and Fig. 3.21, As it is depicted, in the latter figure, based on the exact location of the RSU and calculated distance of the vehicle from the RSU while driven along the path, the curves are overlapping compared to Fig. 3.20 that the minimum values of the rounds are not synchronized. In this scenario, the number of valid measurement rounds is 5 .

The lowest value of the minimum PER values of all the measurement rounds is equal to 0.001 . By comparing antenna selection performance with the SISO case performance, it can be interpreted that although the minimum PER value for both cases is quite close to zero, there is still decrease in PER value when antenna selection is employed. In addition to the minimum PER value, by a more precise look at the results of both cases, it can be seen that the PER curves for the antenna selection have a wider angle than the curves for the SISO case which means for a longer distance, the PER values are at a lower range than in SISO case. By a general look at this figure, it can be interpreted that the trend of the curves looks quite repeatable during all the measurement rounds.

\section{Under Subway Path Scenario}

The third location chosen as measurement track is a direct path located under a subway rail and surrounded by high buildings already shown in Fig. 3.24. The distance from the start point till the end of the path is approximately $280 \mathrm{~m}$ and the RSU is located 


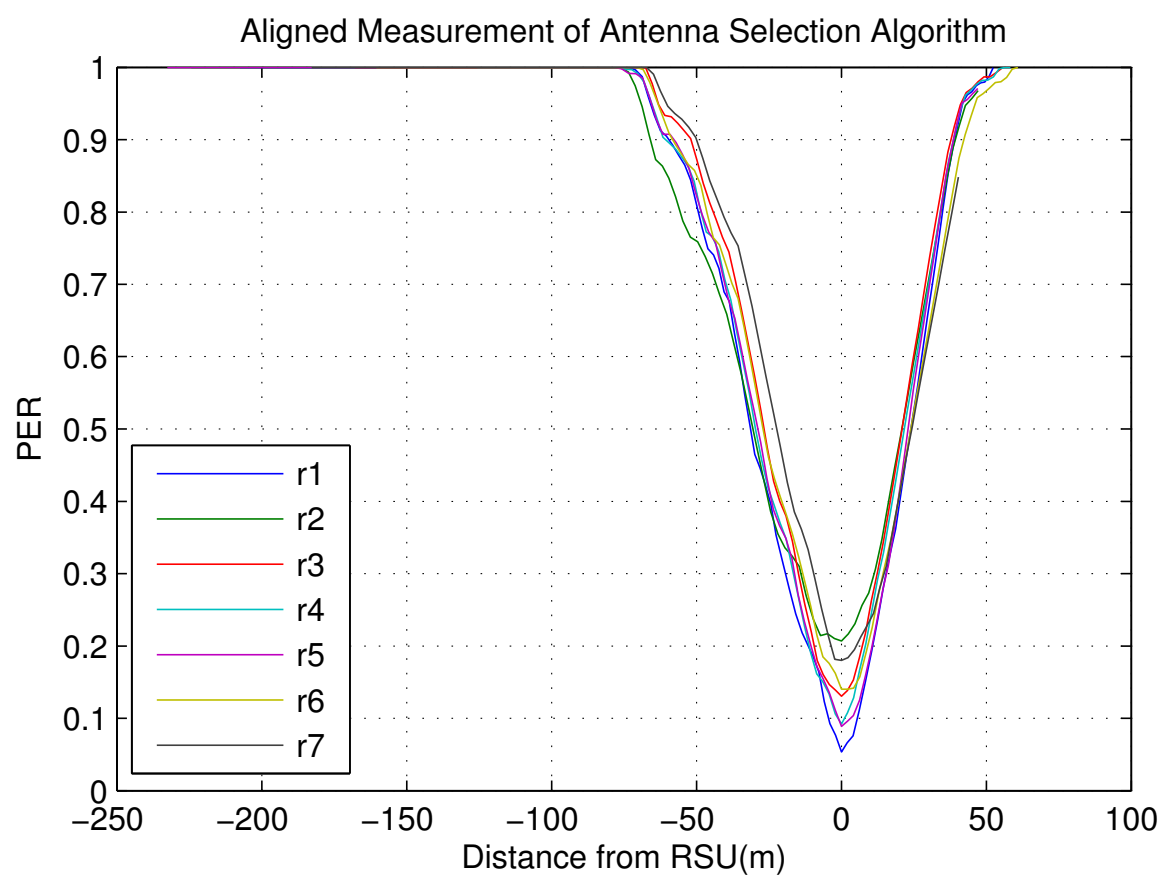

Figure 3.17: Performance evaluation of antenna selection algorithm in direct path scenario - PER vs. distance from RSU

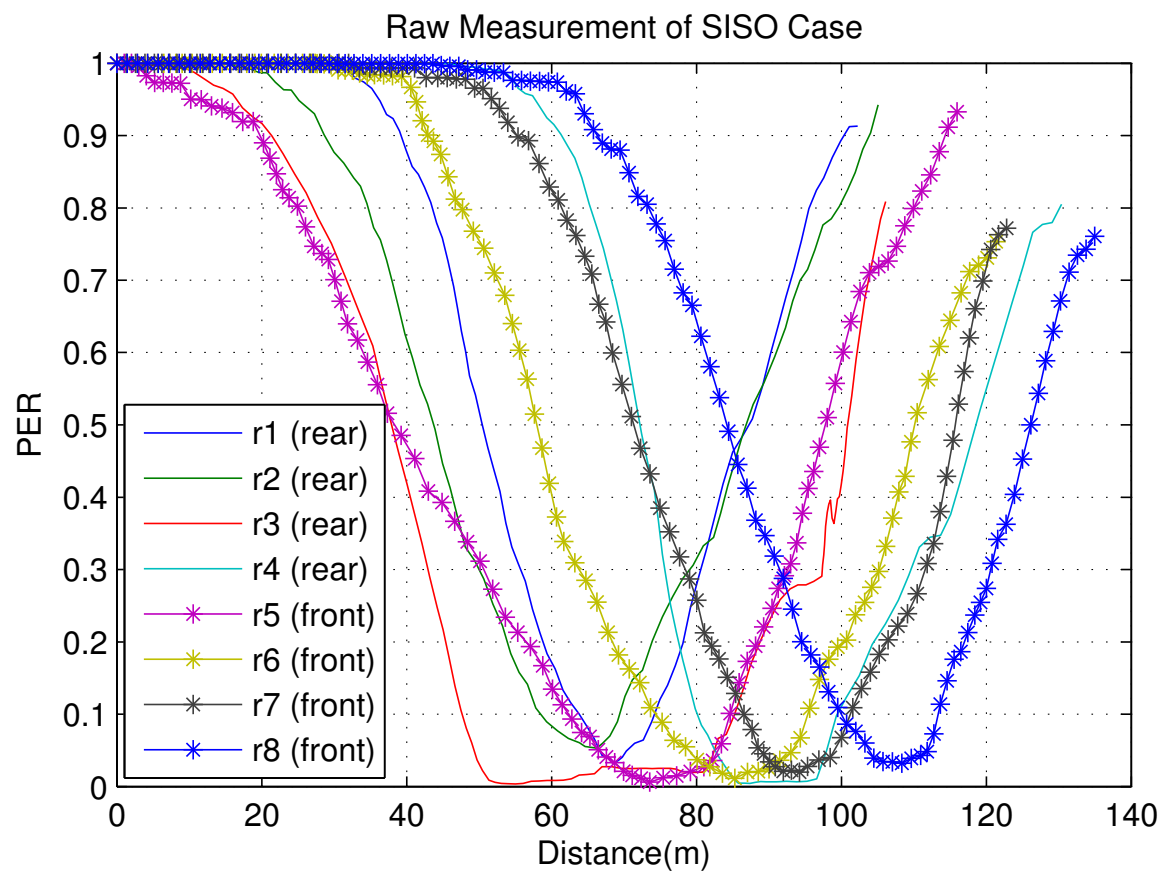

Figure 3.18: Performance evaluation of SISO case in L-Turn scenario - PER vs. total driven distance 


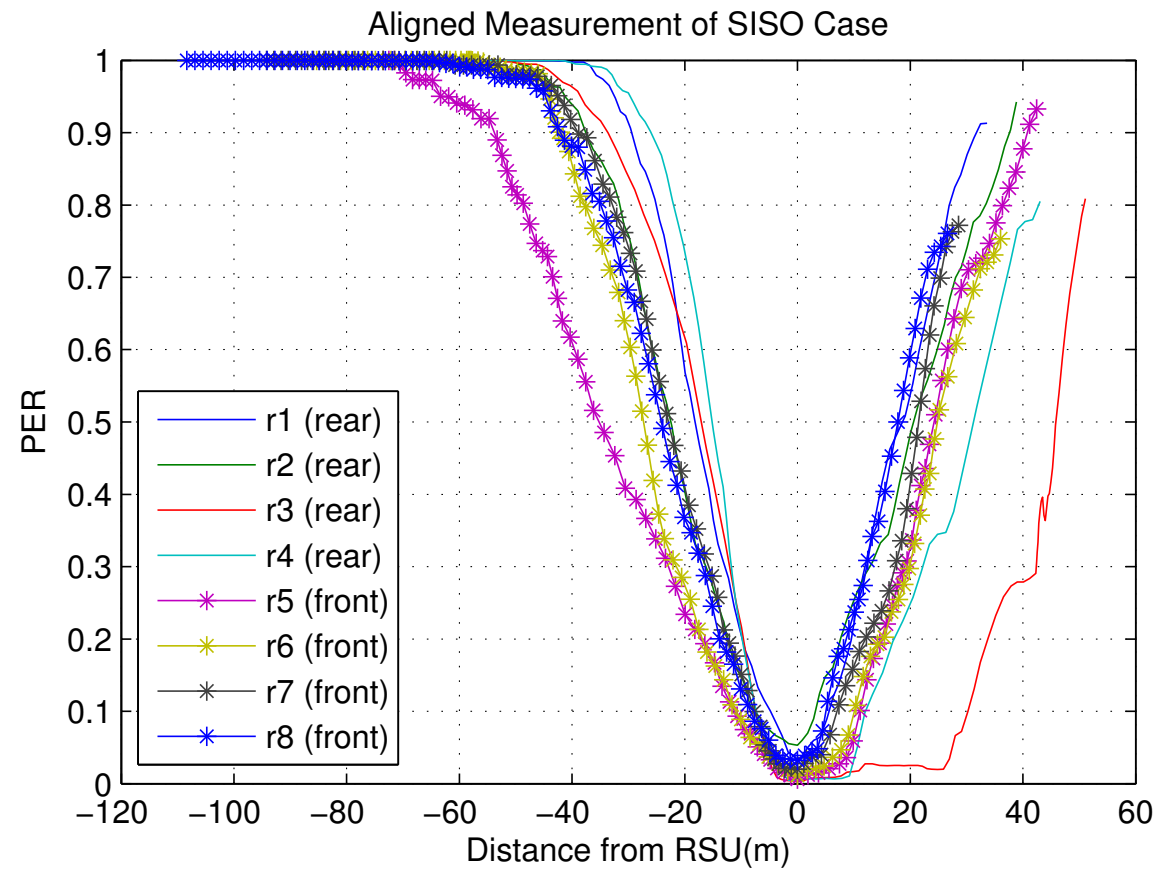

Figure 3.19: Performance evaluation of SISO case in L-Turn scenario - PER vs. distance from RSU

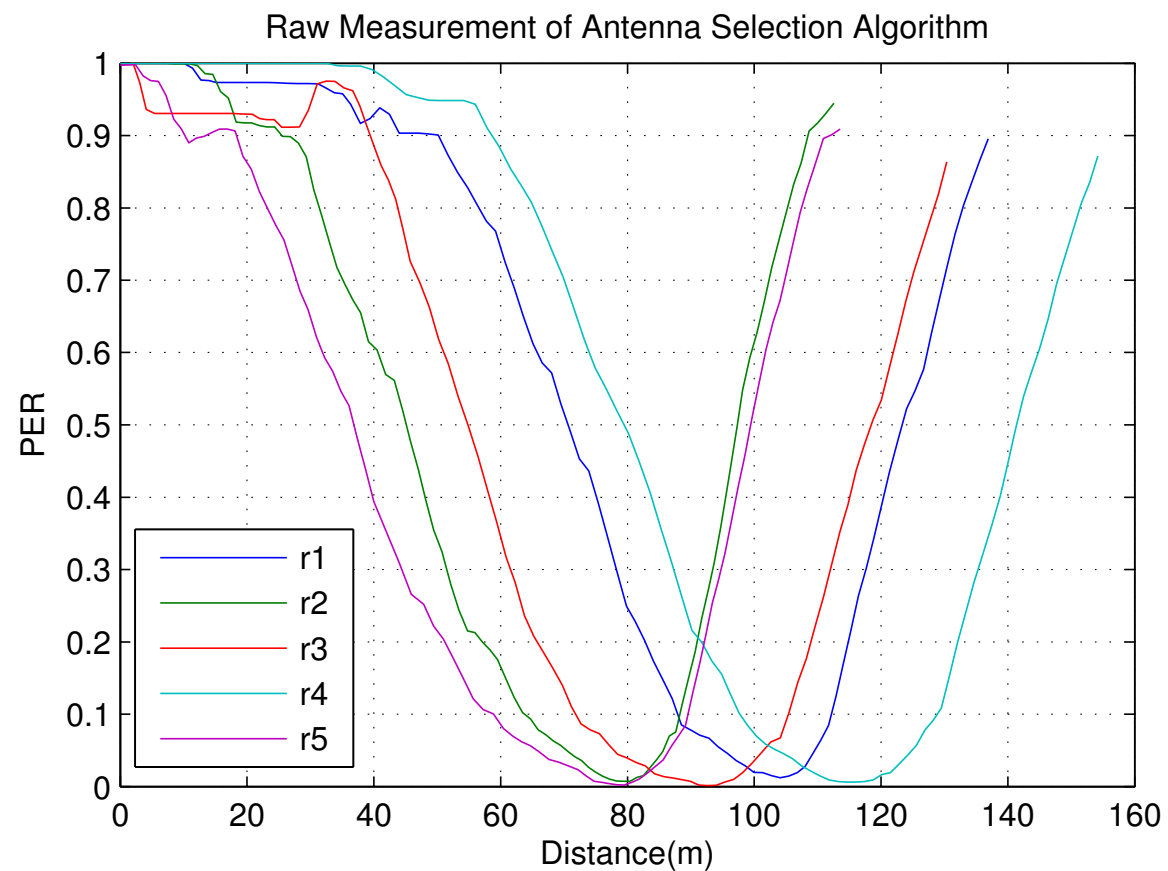

Figure 3.20: Performance evaluation of antenna selection algorithm in L-Turn scenario - PER vs. total driven distance 


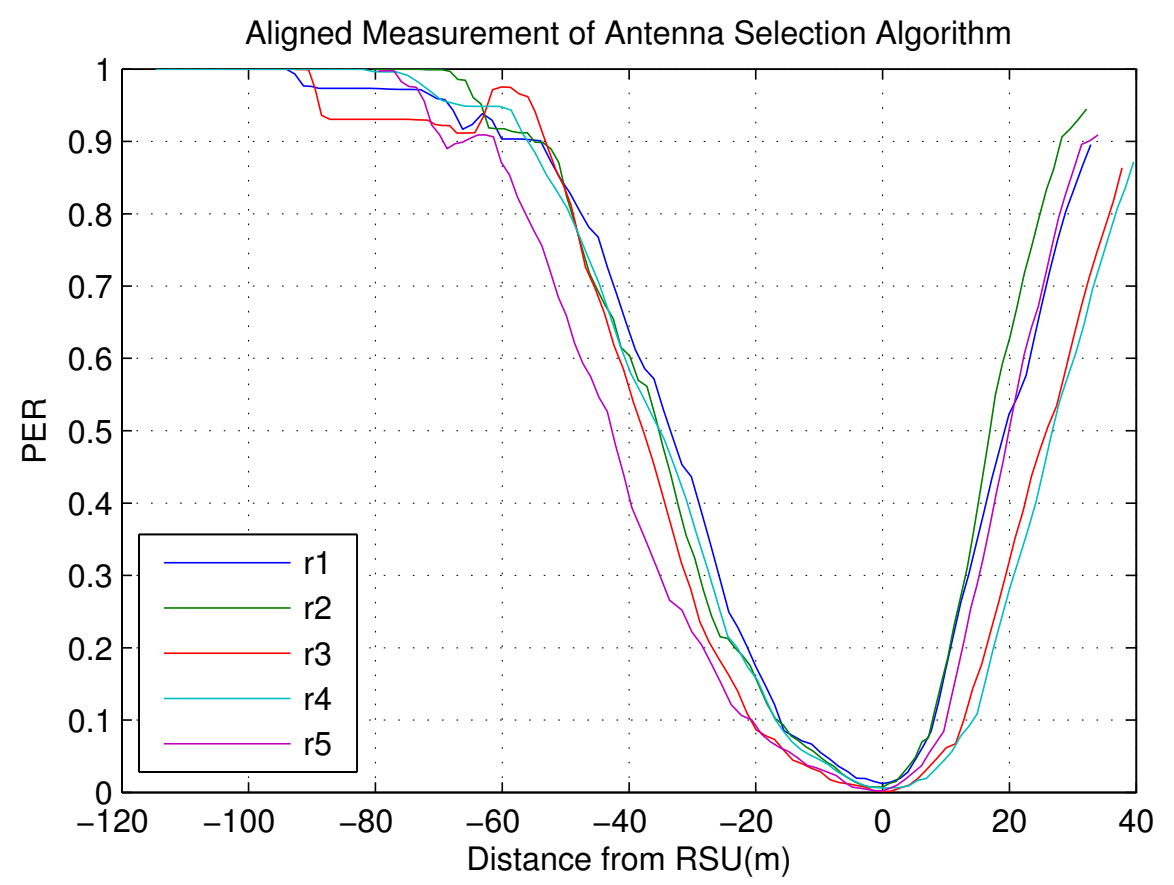

Figure 3.21: Performance evaluation of antenna selection algorithm in L-Turn scenario - PER vs. distance from RSU

on the right side of the path. The driving route is not completely straight since it has a slight curve.

In the first part of the measurement, the SISO case was chosen and eight rounds of measurement were carried out in which the first four rounds were assigned to the rear antenna and in the second four rounds the front antenna was chosen. In Fig. 3.22 and Fig. 3.23, the curves that correspond to the front antenna are solid lines with marks and the rear antenna evaluation results are shown with solid lines. Similar to previous scenarios, the difference between two figures is just the horizontal axis that for the first one, the PER values are plotted based on the total driven distance and for the second figure, the exact location of RSU from GPS data is taken to show the distance of the vehicle before and after the RSU. In this case, among the minimum values of PER, the lowest value for the rear antenna is 0.15 in the first round and for the front antenna, this value is 0.13 in the last round of measurement. As a general result, it can be stated that the PER values lie from 0.13 till 0.26 in this scenario.

Antenna selection algorithm has been employed for the second part of the measurement for the subway scenario. The performance evaluation results are presented in Fig. 3.24 and Fig. 3.25. The number for valid measurement rounds is nine out of total 12 rounds. Based on the calculated PER values, the lowest PER is 0.01 which happens in the first round of measurement. The minimum values of PER has a range of 0.01 till 0.08. Comparing to the SISO case in this scenario, a remarkable improvement has been 


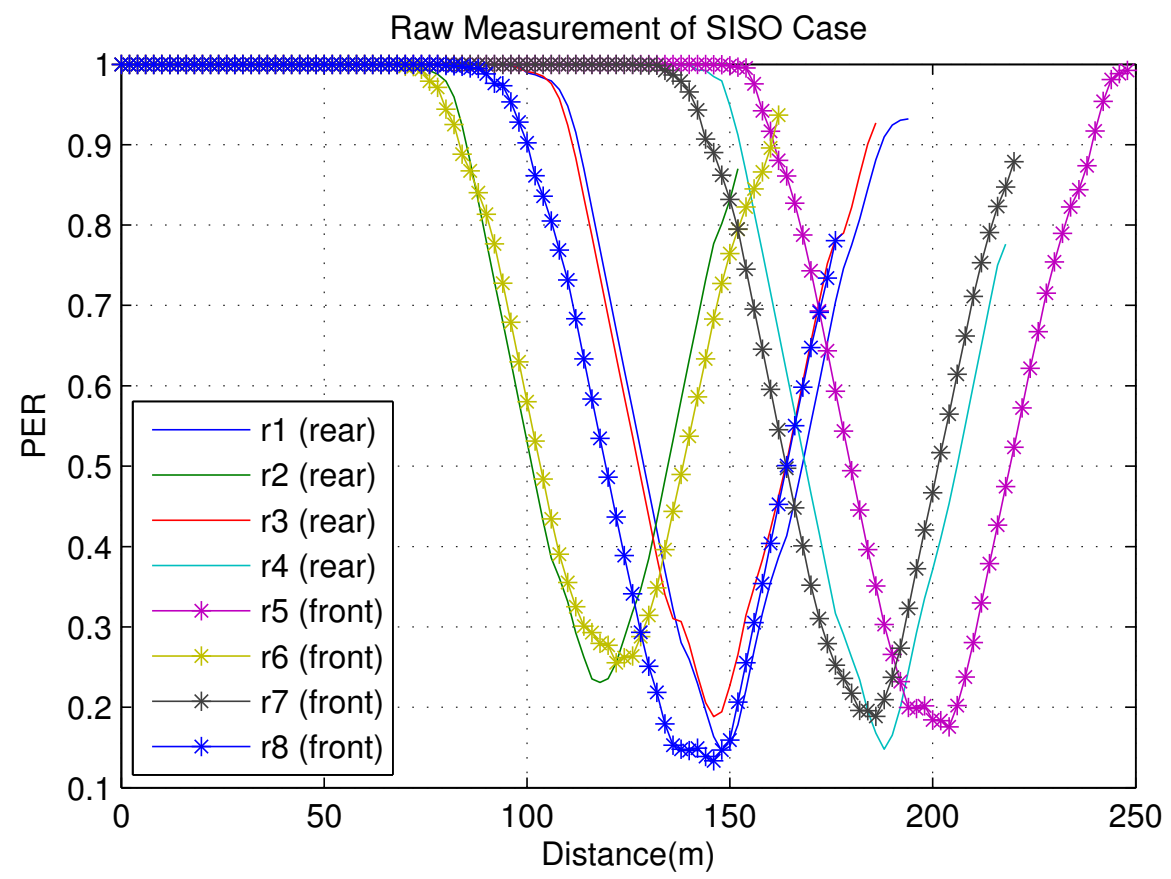

Figure 3.22: Performance evaluation of SISO case in Subway scenario - PER vs. total driven distance

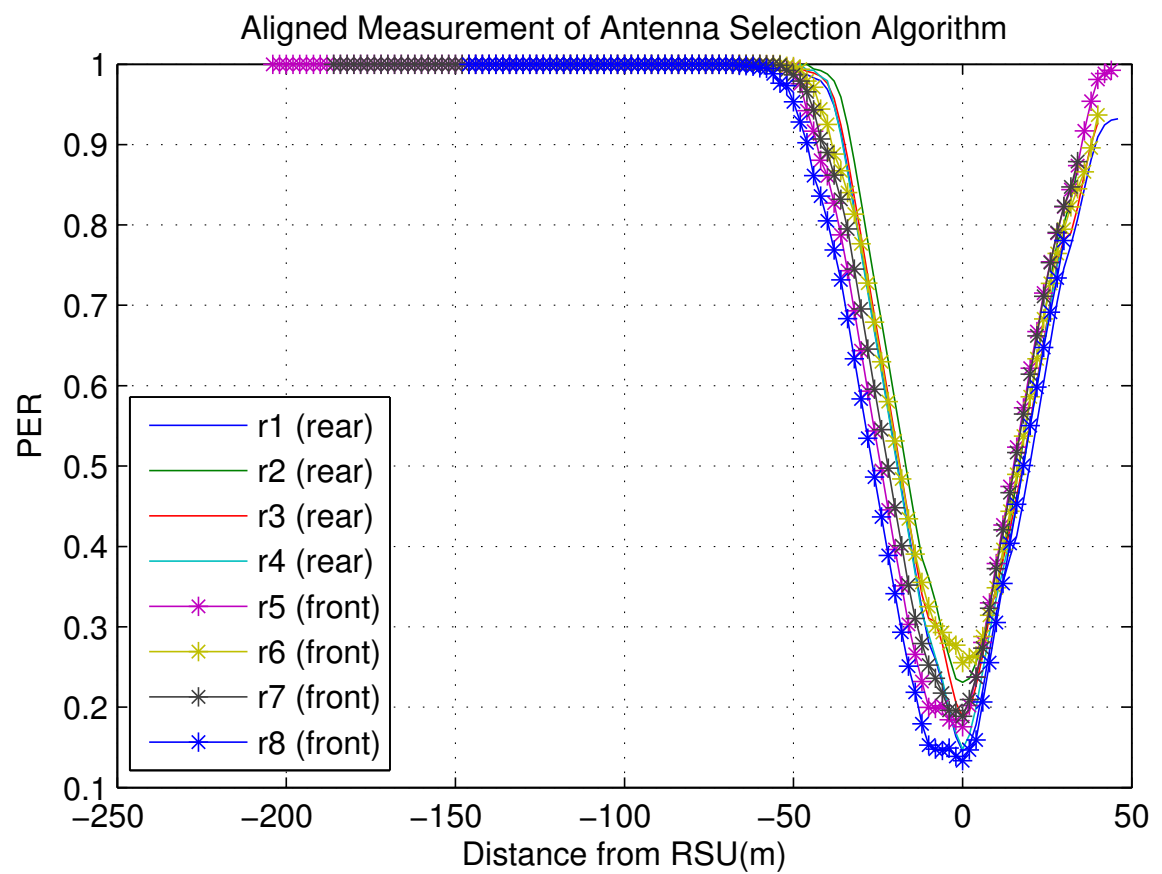

Figure 3.23: Performance evaluation of SISO case in Subway scenario - PER vs. distance from RSU 
achieved by using the antenna selection algorithm in such environment.

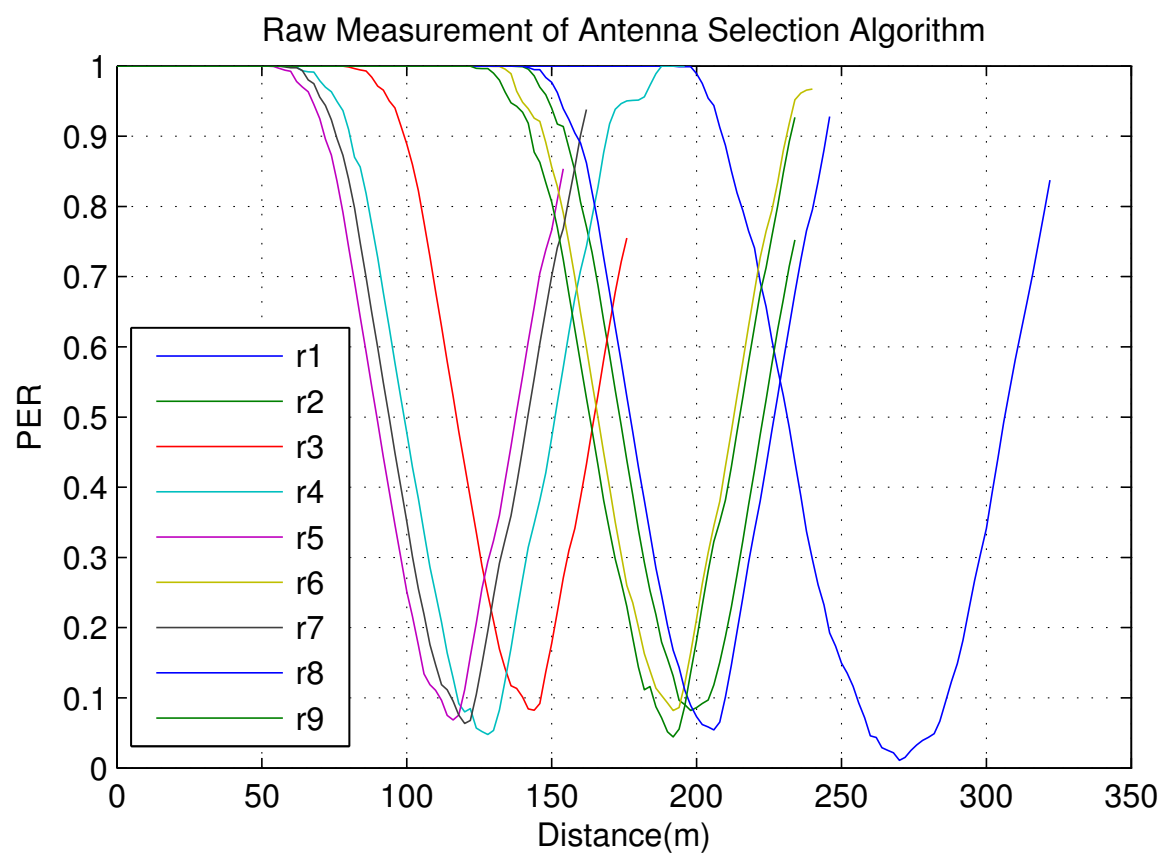

Figure 3.24: Performance evaluation of antenna selection algorithm in Subway scenario - PER vs. total driven distance

\subsection{Discussion and Conclusion}

In this chapter, I have presented the measurement campaign which was done in the urban areas in order to evaluate the performance of my proposed antenna selection algorithm for V2I scenario. Three different locations with different routes and environments have been chosen for the carried out measurement campaigns; Direct path , L-Turn and under subway scenario. In all scenarios the velocity of the vehicle was kept between 20 to $30 \mathrm{~km} / \mathrm{h}$. For each scenario, SISO case and antenna selection algorithm were performed separately in 12 consecutive measurement rounds.

In order to evaluate the performance of the antenna selection diversity in wireless vehicular communications, PER values have been estimated during each round of measurements. The PER results have been shown based on the total driven distance from the starting point to the ending in each scenario and also based on the distance of the moving vehicle from the exact location of RSU. Since the two Rx antennas have been mounted with an angle of $90^{\circ}$ on the roof in a way that the front antenna was pointing to the side of the vehicle and the rear antenna to the front part of it, the SISO case was tested for both antennas, separately. 


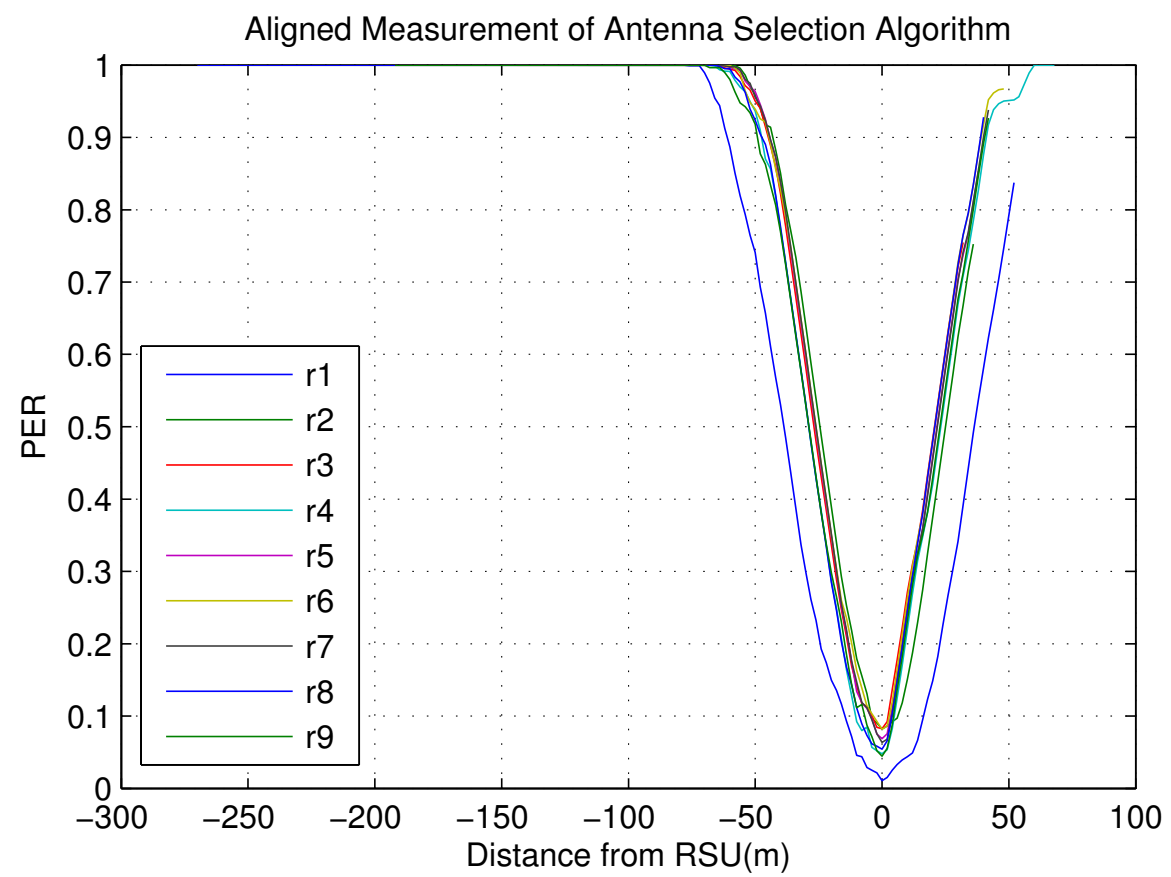

Figure 3.25: Performance evaluation of antenna selection algorithm in Subway scenario - PER vs. distance from RSU

The results of the measurement campaign have confirmed that by employing the antenna selection algorithm, the diversity gain can be improved significantly in a real vehicular environment. Based on the PER values, in the direct path scenario, PER can reach 0.05 in the case the antenna selection algorithm was used while for the SISO case, the lowest minimum PER was 0.22 . In other words, the number of received packets has been increased four times when the antenna selection is used. For the L-Turn scenario, the lowest value among the minimum PER of the rounds has not been changed by using the proposed algorithm; 0.003 to 0.001 . But the average of the minimum values of PER over the measurement rounds was 0.02 for the SISO case and 0.005 for the antenna selection approach. Therefore, in total, it can be said that in this scenario also the diversity gain has been improved by a factor of 0.25 . In the last scenario, there was a remarkable increase in the minimum value of PER. For the SISO case, this value was 0.13 while for the case of the antenna selection algorithm, it was 0.01 . Moreover, among these three scenarios, as also shown in the figures, the PER values are at their lower range due to the longer Line of Sight (LOS) with the Tx antenna in the L-Turn scenario. 



\section{CONCLUSIONS}

Due to the high interest in exploiting MIMO systems in wireless vehicular communications in order to take advantage of the advantages of such system, the hardware cost is imposed because of multiple RF chains. One feasible solution to combat the additional cost is to select a subset of the antenna elements instead of using all elements in each transmit and receive phase. Based on the importance of this issue, the motivation for this work was established and I propose an antenna selection algorithm to improve the BER and higher spatial diversity are achieved. The evaluation of the proposed algorithm has been done via simulations and also a measurement campaign in real environment.

In the first part of my work, in order to develop the proposed algorithm, the frame structure needed to be modified, thus, additional preambles have been added to be used for the selection purpose. Then, the selection itself has been done in three different ways. The first one was the antenna selection algorithm based on the maximum of minimum SNR. In the other two, the channel predictors have been used in order to predict the upcoming frame; Lagrange Interpolation and Linear Regression. By employing theses channel predictors, the channel coefficient is predicted for the preamble different from the four added ones. This preamble is followed by the data symbols in the OFDM frame. After obtaining the predicted channel coefficient, the channel gain is calculated and by using a maxmin function, the receive antenna with better link is selected.

In the second part of my dissertation, the measurement campaign for the antenna selection diversity has been discussed and the performance evaluation results are shown. Similar to the first part, there is a SIMO system consisting of two receive antennas and one transmit antenna. The SNR-based antenna selection algorithm has been tested in three different scenarios in the urban areas of Vienna, Austria.

To sum up my dissertation, I will summarize the key findings of my work and also, highlight some open issues of this dissertation which can be continued and be taken into consideration for further research.

\subsection{Key Findings}

The simulation results indicate that by using the antenna selection algorithm, the BER is improved and in addition, the diversity gain is enhanced comparing to the case where there is a single antenna at both sides of the link. It can be interpreted from the results 
that when the channel predictors are used, the system performance is better than the case that the antenna selection is only based on the estimated SNR. At at an $E_{\mathrm{b}} / N_{0}$ of $12 \mathrm{~dB}$, by using Lagrange Interpolation predictor, BER reaches $0.5 \cdot 10^{-3}$ and with Linear Regression, this value is $0.3 \cdot 10^{-3}$. While for the case that the antenna selection algorithm is SNR-based and no channel predictor is used, BER is $1.4 \cdot 10^{-3}$. Generally, with higher number of iterations of the channel estimation, the slope of the curves can get steeper and consequently, a higher diversity order is achievable. Two types of channel predictors have shown similar performance in my work but it has been illustrated that for higher values of $E_{\mathrm{b}} / N_{0}$ and also with more iterations of the simulation, Linear Regression outperforms Lagrange Interpolation.

Moreover, the antenna selection diversity algorithms yield a higher diversity gain as observed in a measurement campaign in an urban environment. So, in urban areas, in all three locations that the measurement campaign was held, the results validate BER reduction comparing to the SISO case. The only considerable point is that in a scenario such as L-Turn, it was observed that the BER values were not so different for the SISO case and the antenna selection case but it has been confirmed that with the antenna selection, the range of the minimum BER values gets larger when the antenna selection is employed which means that LOS range has been expanded. Moreover, the results are repeatable during several rounds of measurements.

It can be concluded from the above mentioned outputs of this work that using channel predictors improves the system performance and higher gain is achievable. Furthermore, increasing the number of iterations and also the $E_{\mathrm{b}} / N_{0}$, are two effective items in having a more reliable transmission with improved BER.

\subsection{Outlook}

Although, in my dissertation, a new antenna selection algorithm is proposed for wireless vehicular communications which improves the diversity gain in a SIMO system, there are still some open issues in this work that can be added in order to achieve more from such scheme.

As explained before, in this work, two types of channel predictors have been employed in order to have a better antenna selection. There is the possibility to exploit different types of channel predictors, other than the one already used in this work, to evaluate the performance of the antenna selection algorithm with them. By such approach, it would be possible to exploit the channel predictor which has better prediction in a time-variant channel where the channel state information is outdated quickly. The DPS sequences can be used as a predictor. Such sequences are band-limited and energy-concentrated, simultaneously.

Moreover, in the testbed which was used in the measurement campaign, the implemented antenna selection was based on the SNR, in other words, the received signal power. 
Therefore, with more implementations, it would be feasible to evaluate the channel predictors in real scenarios of wireless vehicular communications. 

APPENDICES 



\title{
A LIST OF TERMS AND SYMBOLS
}

\author{
Variable Description
}

\begin{tabular}{ll}
\hline Latin symbols & \\
\hline$B$ & Bandwidth \\
$C R$ & Coding rate \\
$E_{\mathrm{b}}$ & Energy per bit \\
$f$ & Frequency \\
$F$ & Number of frames \\
$f_{c}$ & Carrier frequency \\
$G$ & Cyclic prefix length \\
$H_{n}(t, f)$ & Time-variant frequency response at $n$-th receive antenna \\
$\hat{\mathbf{H}}_{\mathrm{n}}[p, q]$ & Least square estimated channel coefficient \\
$\left|\hat{\mathbf{H}}_{\mathrm{n}}[\mathbf{q}]\right|^{2}$ & Channel gain at $n$-th receive antenna \\
$L$ & Frame length \\
$M$ & Total number of antennas either at transmitter or receiver \\
$M_{r}$ & Total number of receive antennas \\
$M_{t}$ & Total number of transmit antennas \\
$n$ & Receive antenna index \\
$\hat{n}$ & Optimum receive antenna \\
$N$ & Number of subcarriers \\
$N_{\text {add_pre }}$ & Number of added preambles in the extended frame \\
$N_{\mathrm{bpsc}}$ & Number of coded bits per subcarrier \\
$N_{\text {data }}$ & Number of data symbols in a frame \\
$N_{\mathrm{FFT}}$ & Number of FFT points \\
$N_{\mathrm{G}}$ & Number of samples in guard interval \\
$N_{\text {post }}$ & Number of postamble \\
$N_{\text {pre }}$ & Number of preamble \\
$N_{r}$ & Number of selected receive antennas \\
$N_{r p}$ & Number of correctly received packets \\
$N_{t}$ & Number of selected transmit antennas \\
$N_{t p}$ & Total number of transmitted packets \\
$N_{0}$ & Noise power spectral density \\
$p$ & OFDM symbol index \\
$q$ & Subcarrier index \\
$R_{C}$ & Code rate \\
$\hat{S}_{n}[q]$ & Estimated signal power at $n$-th receive antenna \\
&
\end{tabular}




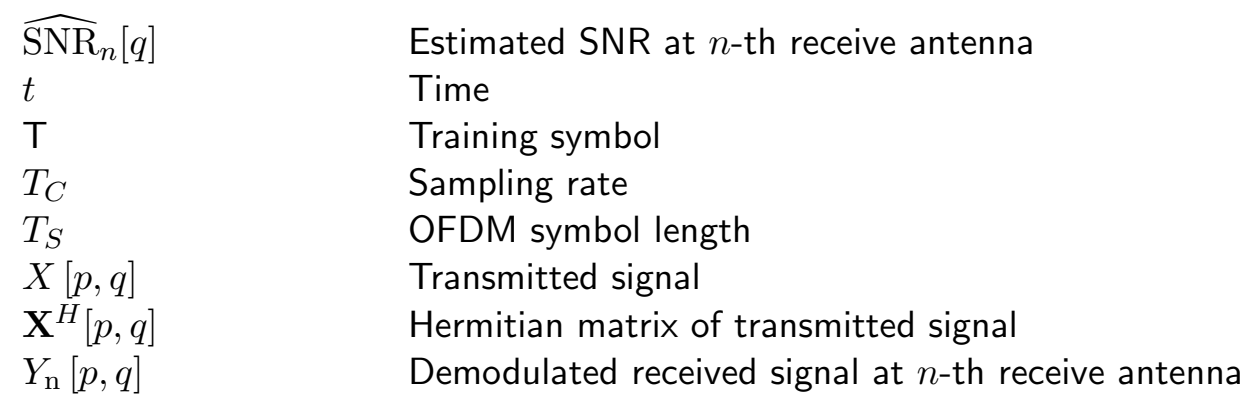

Greek symbols

$\Delta f \quad$ Subcarrier spacing

$\begin{array}{ll}\eta_{\mathrm{n}} & \text { Additive white } \\ \sigma_{N}^{2} & \text { Noise variance }\end{array}$

Operators

$\arg \max _{n}\left(\min _{1 \leq q \leq N}\right) \quad$ minimization over subcarriers and maxmization over receive antenna index $\mathcal{F}^{-1} \quad$ Inverse Fourier Transform

$\mathbf{X}^{H} \quad$ Hermitian transpose of matrix $\mathbf{X}$ 


\section{B LIST OF ACRONYMS}

$\begin{array}{ll}\text { AGC } & \text { Automatic Gain Control } \\ B E R & \text { Bit Error Ratio } \\ B P S K & \text { Binary Phase-shift Keying } \\ C C A & \text { Clear Channel Assessment } \\ C I C A S & \text { Cooperative Intersection Collision Avoidance System } \\ C O T S & \text { Commercial off-the-shelf } \\ C R C & \text { Cyclic Redundancy Check } \\ C S I & \text { Channel State Information } \\ D P S & \text { Discrete Prolate Spheroidal } \\ D S S S & \text { Driving Safety Support System } \\ E G C & \text { Equal Gain Combining } \\ F F T & \text { Fast Fourier Transform } \\ F P G A & \text { Field-programmable Gate Array } \\ G I & \text { Guard Interval } \\ G P S & \text { Global Positioning System } \\ I C P & \text { Interface Communication Protocol } \\ I F F T & \text { Inverse Fast Fourier Transform } \\ I F T & \text { Inverse Fourier Transform } \\ I T S & \text { Intelligent Transport System } \\ I V H S & \text { Intelligent Vehicle Highway Systems } \\ L T E & \text { Long-Term Evolution } \\ L O S & \text { Line of Sight } \\ L S & \text { Least Square } \\ M A C & \text { Media Access Control } \\ M I M O & \text { Multiple-input multiple-output } \\ M R C & \text { Maximal Ratio Combining } \\ O F D M & \text { Orthogonal Frequency Division Multiplexing } \\ P E R & \text { Packet Error Ratio } \\ P H Y & \text { Physical Layer } \\ P L C P & \text { Physical Layer Convergence Protocol } \\ P S D U & \text { Physical Layer Service Data Unit } \\ Q A M & \text { Quadrature Amplitude Modulation } \\ Q P S K & \text { Quadrature Phase Shift Keying } \\ R F & \text { Radio Frequency } \\ R I O & \text { Reconfigurable Input/Output } \\ R S U & \text { Road Side Unit } \\ R x & \text { Receive, Receiver } \\ & \end{array}$




$\begin{array}{ll}\text { SDR } & \text { Software Defined Radio } \\ \text { SIMO } & \text { Single-input multiple-output } \\ \text { SISO } & \text { Single-input single-output } \\ \text { SNR } & \text { Signal to Noise Ratio } \\ \text { TX } & \text { Transmit, Transmitter } \\ \text { USRP } & \text { Universal Software Radio Peripheral } \\ \text { VANET } & \text { Vehicular Adhoc Network } \\ \text { V2I } & \text { Vehicle-to-infrastructure } \\ \text { V2V } & \text { Vehicle-to-vehicle } \\ \text { V2X } & \text { Vehicle-to-vehicle/infrastructure } \\ \text { VRLA } & \text { Valve Regulated Lead Acid }\end{array}$




\section{MEASUREMENT CAMPAIGN TESTBED}

This appendix documents used equipment in the measurement campaign and much materials are taken from [6], 7] and [32].

\section{C.1 PXle-1082}

The NI PXle-1082 is an eight-slot chassis which has a high-bandwidth backplane with PXI Express capability in each of its slots so that it can fulfill the requirement for a wide range of high-performance test and measurement applications such as high-speed measurements, data streaming and high-channel-density system solutions. Additionally, the PXle-1082 is compatible with both PXI Express and also CompactPCI Express modules. The eight PXI hybrid-compatible slots consist of one PXI Express system slot and seven peripheral slots. Furthermore, there are a total number of nine PXI Express slots.

Fig. C.1] shows the overview of the PXle-1082 backplane [32].

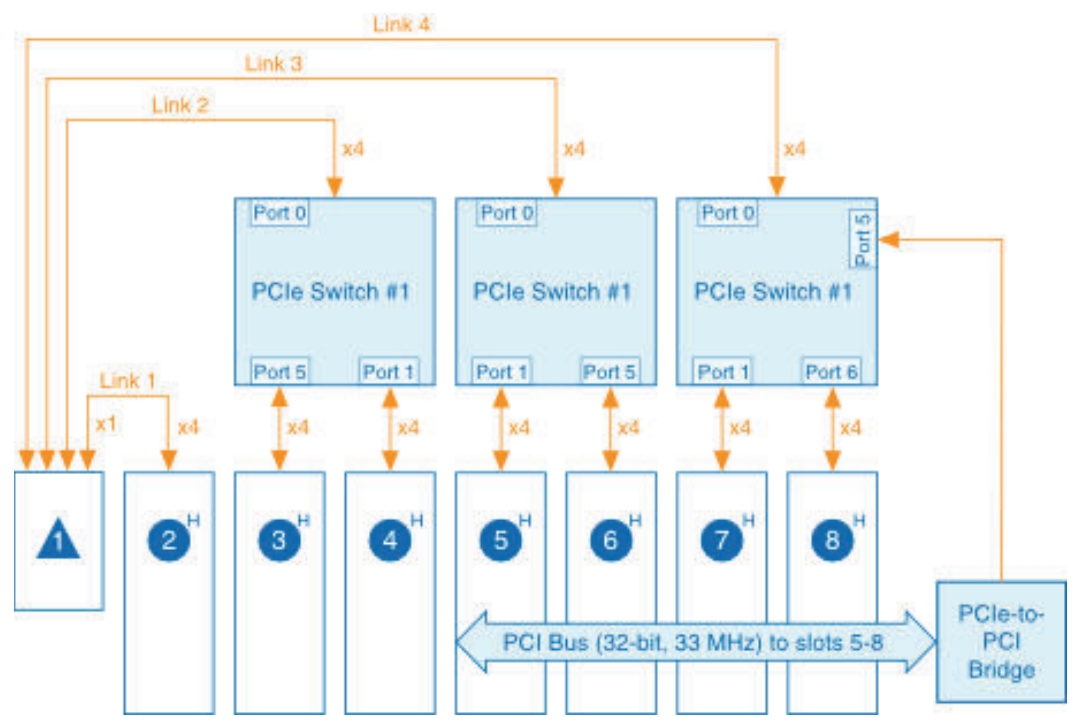

Figure C.1: PXle-1082 Backplane Overview 
The advantage of the PXle-1082 is first the high reliability including an extended temperature range which is 0 to $55{ }^{\circ} \mathrm{C}$ and a total power of $420 \mathrm{~W}$ available for such temperature range. In order to have higher reliability, the devices are HALT-tested. The second advantage is the multichassis synchronization which provides different synchronization options like CLK10 sharing, GPS, IEEE 1588, IRIG-B and etc. Last but not least is portable LCD monitor and keyboard accessories and also front and rear rack-mount kits.

In the following figures, the PXle-1082 chassis dimensions are shown. In Fig. C.2 in the upper figure, the dimensions of the chassis are shown from the front view while in the lower part of this figure, the dimensions are from the side view.

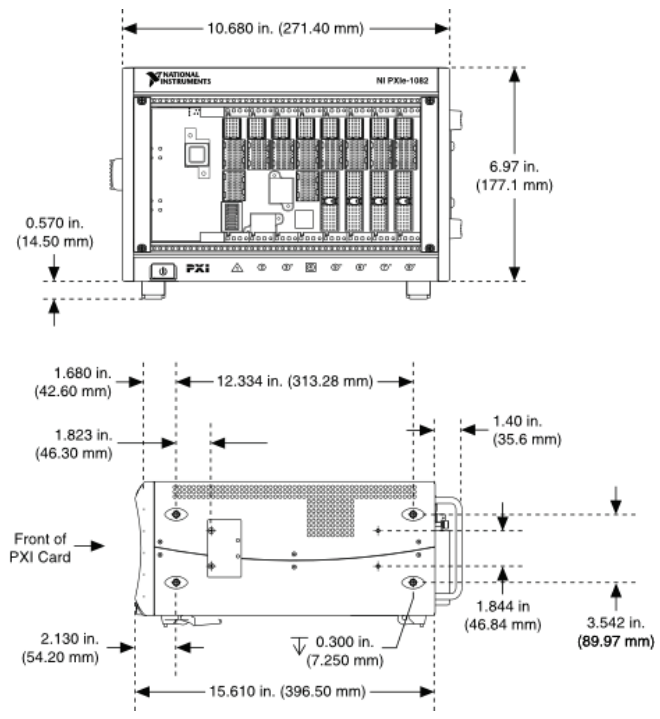

Figure C.2: PXle-1082 Chassis Dimensions (front and side)

Fig. C.3 illustrates the dimensions of this chassis from the bottom view.

The rack mount kit components are shown in Fig. C.4. In this figure, component no. 1 is the front rack mount kit, no. 2 shows the PXle-1082 chassis and no. 3 is the rear rack mount kit.

The backplane illustrated in Fig. C.1, is completely compliant with PXI timing and synchronization standards. A $10 \mathrm{MHz}$ reference clock with an accuracy of \pm 25 parts per million (ppm), less than $5 \mathrm{ps}$ jitter, and a maximum slot-to-slot skew of $300 \mathrm{ps}$. In case triggering or handshaking is required, the PXI trigger bus and PXI star trigger are provided by the PXle-1082.

There is a differential $100 \mathrm{MHz}$ reference clock included for the PXI Express modules with an accuracy of $\pm 25 \mathrm{ppm}$, less than $3 \mathrm{ps}$ jitter and a maximum slot-to-slot skew of 


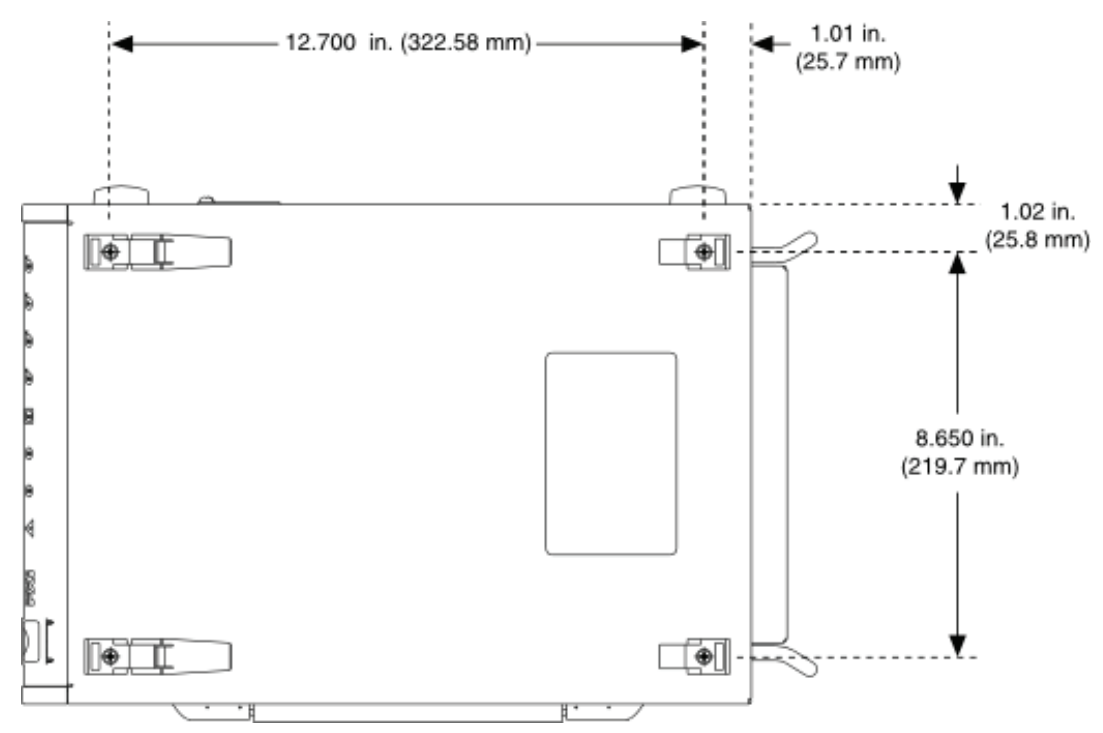

Figure C.3: PXle-1082 Chassis Dimensions (bottom)

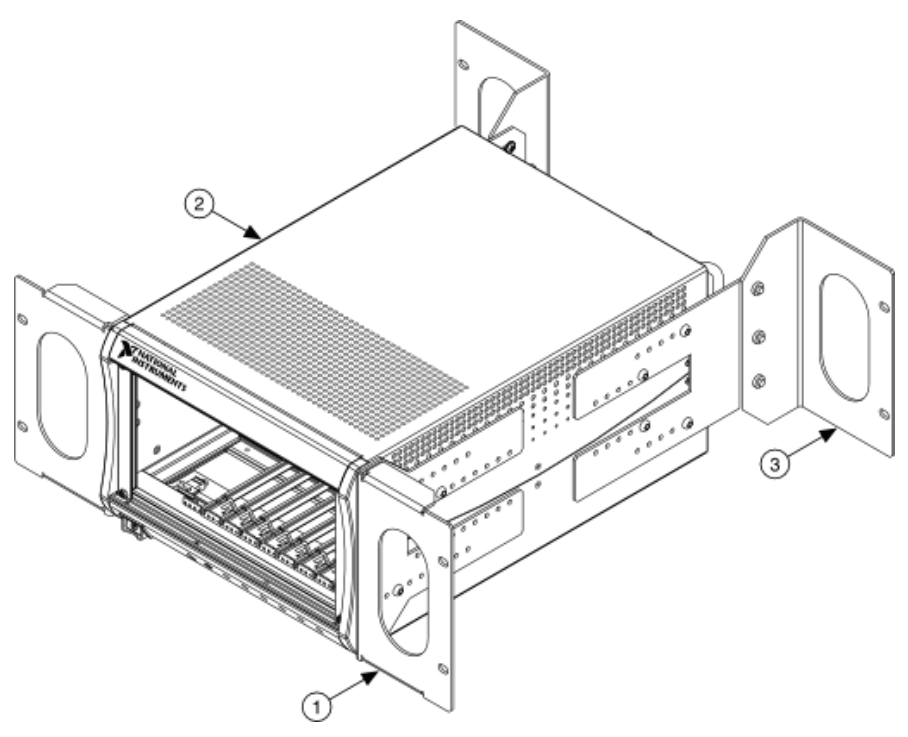

Figure C.4: PXle-1082 Chassis Rack Mount Kit Components 
100 ps. In order to deliver an intermodule skew less than 200 ps, there is a differential star trigger to the PXI Express slots.

\section{C.2 USRP-2953R}

The USRP RIO software defined radio platform with an integrated hardware and software can be a solution for high-performance wireless communication systems. In each USRP RIO device, there are two RF transceivers and a large Xilinx Kintex-7 (410T) FPGA which is a reconfigurable LabVIEW FPGA target that incorporates DSP48 coprocessing for high-rate, low-latency applications. Based on the mentioned features, this design flow can be used for faster prototyping in many advanced research topics including MIMO systems, LTE relaying, spectrum sensing cognitive radio, beam-forming and synchronization of heterogeneous networks.

USRP-2953R is a $2 \times 2$ MIMO RF transceivers which can be independently tuned from $1.2 \mathrm{GHz}$ to $6 \mathrm{GHz}$ and the real time bandwidth for each channel is $40 \mathrm{MHz}$ or $120 \mathrm{MHz}$. There is the possibility to expand the MIMO system to higher channel count MIMO systems. The USRP RIO is also unified with LabVIEW design flow for the host and FPGA and additionally compatible with the LabVIEW Communications System Design Suite. The RF performance has been optimized for the purposed of using the LabVIEW Communications System Design Suite and LabVIEW FPGA module including corrections for DC offset, I/Q imbalance and amplitude accuracy.

USRP RIO can be employed in different applications and technologies such as massive MIMO, waveform prototyping, 5G PHY/MAC research, cell network/NFC, dynamic spectrum access, RADAR prototyping and detection and cognitive radio.

Fig. C.5 presents the block diagram of a USRP RIO. As shown in this figure, there are two independent RF transceivers and the corresponding ports which can support a $2 \times 2$ MIMO system. The USRP RIO is equipped with a high-speed, low-latency PCI Express $x 4$ connection to the host which can be a desktop, laptop or a PXI chassis where the LabVIEW communications Design Suite is accessible and consequently a wide variety of IP for RF and wireless communications can be implemented for the host or the FPGA with the functions and reference designs.

A typical USRP RIO can be found in Fig. C.6 which shows the system components. As mentioned above, in addition to a host computer, an MXI-Express $\times 4$ interface card and also a cable to connect the USRP RIO device are required.

LabVIEW-based graphical programming presents integrated analysis functions for various signal processing and communications techniques and therefore, a large area of applications can be included to be implemented on the host side or on the FPGA. Furthermore, LabVIEW Communications Design Suite can provide text-based supports with the text integration nodes. Thus, any kind of input file such as .m code and $\mathrm{C}$ code can be easily imported in order to prototype the existing algorithm on the USRP hardware. 


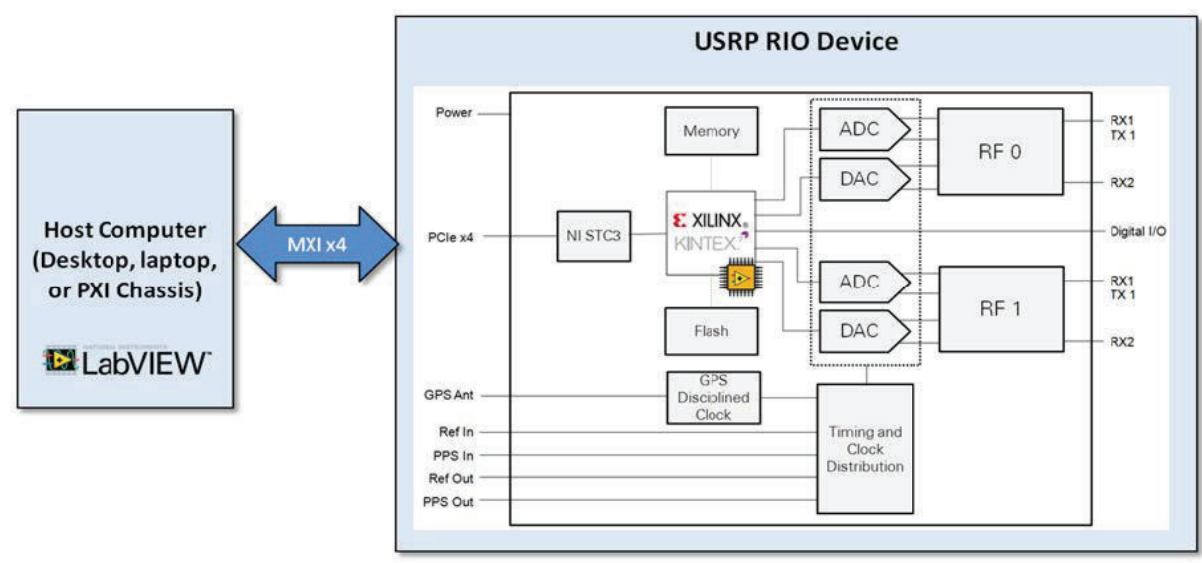

Figure C.5: USRP RIO Block Diagram

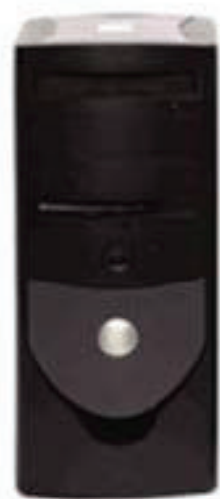

PC

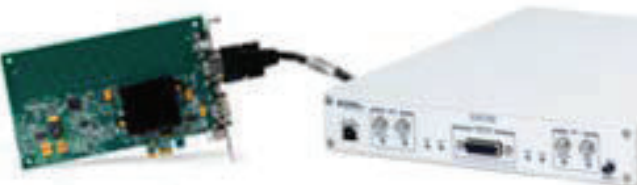

MXle Adapter
NI USRP-RIO

Figure C.6: USRP RIO System Component 



\section{BIBLIOGRAPHY}

[1] P. Papadimitratos, A. Fortelle, K. Evenssen, R. Brignolo, and S. Cosenza, "Vehicular Communication Systems: Enabling Technologies, Applications, and Future Outlook on Intelligent Transportation," IEEE Communications Magazine, vol. 47, pp. 84-95, 2009.

[2] A. N. Shahab Sanayei, "Adaptive antennas and mimo systems for wireless communications antenna selection in mimo systems."

[3] M. Shemshaki, T. Zemen, and C. Mecklenbräuker, "Antenna selection diversity for IEEE 802.11p," in Industrial Electronics Society, IECON 2013 - 39th Annual Conference of the IEEE, Wien, November 2013, pp. 6876-6879.

[4] T. Zemen, L. Bernado, N. Czink, and A. F. Molisch, "Iterative time-variant channel estimation for $802.11 \mathrm{p}$ using generalized discrete prolate spheroidal sequences," vol. 61, no. 3, pp. 1222-1233, 2012.

[5] M. Shemshaki and C. Mecklenbräuker, "Antenna Selection Algorithm with Improved Channel Predictor for Vehicular Environment," in IEEE 6th International Symposium on Wireless Vehicular Communications (WiVeC), 2014.

[6] "USRP RIO - Software Defined Radio," National Instruments, Tech. Rep., 2014.

[7] "8-Slot PXI Express Chassis for PXI and PXI Express Modules - NI PXle-1082," National Instruments, Tech. Rep., 2014.

[8] L. Le, A. Festag, and R. Baldessari, "Vehicular Wireless Short-range Communication for Improving Intersection Safety," IEEE Communications S, vol. 47, pp. 104-110, 2009.

[9] M. Emmelmann, B. Bochow, and C. C. Kellum, Vehicular Networking: Automotive applications and beyond. John Wiley \& Sons Ltd., 2010.

[10] K. H. Chang, "Wireless Communications for Vehicular Safety," IEEE Wireless Communications, vol. 22, pp. 6-7, 2015.

[11] L. Cheng, B. E. Henty, D. D. Stancil, F. Bai, and P. Mudalige, "Mobile Vehicle-toVehicle Narrow-Band Channel Measurement and Characterization of the $5.9 \mathrm{GHz}$ Dedicated Short Range Communication (DSRC) Frequency Band," IEEE Journal on Selected Areas in Communications, vol. 25, pp. 1501-1516, 2007. 
[12] A. F. Molisch, F. Tufvesson, J. Karedal, and C. Mecklenbräuker, "A Survey on Vehicle-to-vehicle Propagation Channels," IEEE Wireless Communications, vol. 16, pp. 12-22, 2009.

[13] C. Mecklenbräuker, A. F. Molisch, J. Karedal, F. Tufvesson, A. Paier, L. Bernado, T. Zemen, O. Klemp, and N. Czink, "Vehicular Channel Characterization and Its Implications for Wireless System Design and Performance," Proceedings - IEEE, vol. 99, pp. 1189-1212, 2011.

[14] K. Furstenberg, "A New European Approach for Intersection Safety - The ECProject INTERSAFE," in IEEE Conference on Intelligent Transportation System, 2005.

[15] M. Y. et al., "Development of Vehicle-Infrastructure Cooperative Systems-Field Operational Test of Hiroshima DSSS," in ITS World Congress, 2008.

[16] "Cooperative Intersection Collision Avoidance Systems," http://www.its.dot.gov/cicas/.

[17] M. J. G. G. J. Foschini, "On limits of wireless communications in a fading environment when using multiple antennas," 1998.

[18] J. Winters, "On the Capacity of Radio Communication Systems with Diversity in a Rayleigh Fading Environment," IEEE Journal on Selected Areas in Communications, vol. 5, pp. 871-878, 1987.

[19] E. Telatar, "Capacity of Multi-antenna Gaussian Channels," European Transactions on Telecommunications, vol. 10, pp. 585-595, 1999.

[20] A. F. Molisch and M. Z. Win, "MIMO systems with antenna selection," IEEE Microwave Magazine, vol. 5, pp. 46-54, 2004.

[21] E. Biglieri, R. Calderbank, A. Constantinides, A. Goldsmith, A. Paulraj, and H. V. Poor, MIMO Wireless Communications. Cambridge, 2007.

[22] A. Attia, A. A. ElMoslimany, A. El-Keyi, T. ElBatt, F. Bai, and C. Saraydar, "MIMO Vehicular Networks: Research Challenges and Opportunities," Journal of Communications, vol. 7, pp. 500-513, 2012.

[23] Y. Gao and T. Kaiser, "Antenna Selection in Massive MIMO Systems: Full-array Selection or Subarray Selection?" in IEEE Sensor Array and Multichannel Signal Processing Workshop (SAM), 2016.

[24] H. G. Engler and E. A. Jorswieck, "Long-term Antenna Selection for Large-scale MIMO Links," in IEEE Sensor Array and Multichannel Signal Processing Workshop (SAM), 2016.

[25] H. A. A. Saleh, A. F. Molisch, T. Zemen, S. D. Blostein, and N. B. Mehta, "Receive Antenna Selection for Time-Varying Channels Using Discrete Prolate Spheroidal Sequences," IEEE Transactions on Wireless Communications, vol. 11, pp. 26162627, 2012. 
[26] R. G. Stephan, C. R. Murthy, and M. Coupechoux, "A Markov Decision Theoretic Approach to Pilot Allocation and Receive Antenna Selection," IEEE Transactions on Wireless Communications, vol. 12, pp. 3813-3823, 2013.

[27] S. Bandopadhaya and K. K. Gupta, "Adaptive Antenna Selection Technique for MIMO System," in Internationl Conference on Signal Processing and Integrated Networks (SPIN), 2016.

[28] S. Prakash and I. McLoughlin, "Predictive Transmit Antenna Selection with Maximal Ratio Combining," in IEEE Global Telecommunications Conference (GLOBECOM), 2009.

[29] Z. Chen, J. Yuan, and B. Vucetic, "Analysis of Transmit Antenna Selection/Maximal-Ratio Combining in Rayleigh Fading Channels," IEEE TranIEEE Transactions on Vehicular Technologies, vol. 54, pp. 1312-1321, 2005.

[30] E. Kurniawan, A. S. Madhukumar, and F. Chin, "Antenna selection technique for MIMO-OFDM systems over frequency selective rayleigh fading channel," IET Communications, vol. 1, no. 3, pp. 458-463, 2007.

[31] R. H. Clarke, "A statistical theory of mobile-radio reception," Bell System Technical Journal, p. 957, 1968.

[32] LabVIEW Communications System Suite 1.0 Manual. 